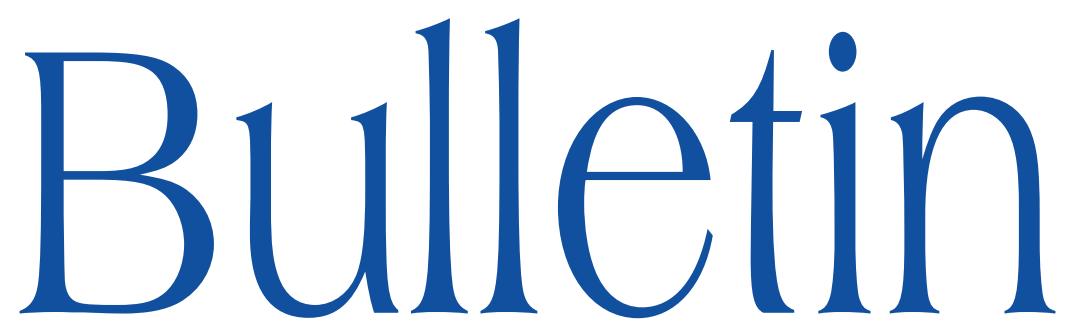

de la SOCIÉTÉ MATHÉMATIQUE DE FRANCE

\title{
THE TANGENT COMPLEX TO THE BLOCH-SUSLIN COMPLEX
}

\author{
Jean-Louis Cathelineau
}

Tome 135

Fascicule 4

2007

SOCIÉTÉ MATHÉMATIQUE DE FRANCE

Publié avec le concours du Centre national de la recherche scientifique 
Bull. Soc. math. France

135 (4), 2007, p. 565-597

\title{
THE TANGENT COMPLEX TO THE BLOCH-SUSLIN COMPLEX
}

\author{
By JeAn-Louis CATHELineau
}

\begin{abstract}
Motivated by a renewed interest for the "additive dilogarithm" appeared recently, the purpose of this paper is to complete calculations on the tangent complex to the Bloch-Suslin complex, initiated a long time ago and which were motivated at the time by scissors congruence of polyedra and homology of $\mathrm{SL}_{2}$. The tangent complex to the trilogarithmic complex of Goncharov is also considered.
\end{abstract}

RÉSUmÉ (Le complexe tangent au complexe de Bloch-Suslin). - À la suite de travaux récents sur le "dilogarithme additif », on se propose de compléter une étude du complexe tangent au complexe de Bloch-Suslin, initiée il y a plus de vingt ans en rapport avec le troisième problème de Hilbert et l'homologie de $\mathrm{SL}_{2}$. On considère aussi le complexe tangent au complexe trilogarithmique de Goncharov.

\section{Introduction}

This paper could have been also entitled: "Infinitesimal algebra of the Abel 5-term relation of the dilogarithm". Our point of view is rather elementary: we complete calculations initiated a long time ago in [6], which were motivated by scissors congruences of polyedra in Euclidean three space. The subject has attracted recently new interest (see specially for another less naive point

Texte reçu le 7 juin 2007, accepté le 21 février 2008

Jean-Louis Cathelineau, Laboratoire Jean Dieudonné UMR CNRS 6621, Parc Valrose 06108 Nice Cedex 2 • E-mail : cathe@math.unice.fr

2000 Mathematics Subject Classification. - 11G55, 14F42, $19 \mathrm{E} 15$.

Key words and phrases. - Bloch-Suslin complex, additive dilogarithm, tangent functors. 
of view, the work of Bloch-Esnault in [3]) and is related to many questions, namely (here $k$ is a field)

- Homology of $\mathrm{SL}_{2}(k)$ with coefficients in the adjoint action and its exterior powers over $\mathbb{Z}[6,12]$,

- Relative algebraic $K$-theory and relative cyclic homology of dual numbers $k[\varepsilon][7,11]$,

- Scissors congruences of polyedra in Euclidean spaces [9, 17, 5],

- Infinitesimal polylogarithms [8, 13],

- Cycles with modulus and additive polylogarithms [4, 3, 27, 26, 28],

- The possible existence of a theory of mixed Tate motives over dual numbers [16, 17, 3],

- Tangent spaces to spaces of algebraic cycles and formal deformations of Chow groups [20, 21].

Let the Bloch-Suslin complex of a field be the cohomological complex in degree 1 and 2

$$
D: \mathcal{B}(k) \rightarrow \bigwedge^{2} k^{\times}
$$

where $\mathcal{B}(k)$ is the commutative group with generators symbols $\{a\}$, where $a \in$ $k^{\times}$and $a \neq 1$, submitted to the five terms relation

$$
\{a\}-\left\{a^{\prime}\right\}+\left\{\frac{a^{\prime}}{a}\right\}-\left\{\frac{1-a^{\prime}}{1-a}\right\}+\left\{\frac{a\left(1-a^{\prime}\right)}{a^{\prime}(1-a)}\right\}=0,
$$

and

$$
D(\{a\})=a \wedge(1-a) .
$$

When $k$ is algebraically closed of characteristic 0 , this complex can be inserted into several exact sequences (see Suslin [31] for finer results): the Bloch-Wigner sequence [10]

$$
0 \rightarrow \mu(k) \rightarrow H_{3}\left(\mathrm{SL}_{2}(k), \mathbb{Z}\right) \rightarrow \mathcal{B}(k) \rightarrow \bigwedge^{2} k^{\times} \rightarrow H_{2}\left(\mathrm{SL}_{2}(k), \mathbb{Z}\right) \rightarrow 0
$$

or its variant in terms of algebraic $K$-theory groups

$$
0 \rightarrow \mu(k) \rightarrow K_{3}^{\text {ind }}(k) \rightarrow \mathcal{B}(k) \rightarrow \bigwedge^{2} k^{\times} \rightarrow K_{2}(k) \rightarrow 0 .
$$

This last sequence, tensored by $\mathbb{Q}$, can be rewritten

$$
0 \rightarrow K_{3}^{(2)}(k) \rightarrow \mathcal{B}(k) \otimes \mathbb{Q} \rightarrow \bigwedge^{2} k^{\times} \rightarrow K_{2}^{(2)}(k) \rightarrow 0,
$$

where the $K_{n}^{(i)}(k)$ are the pieces of the Adams decomposition of $K_{n}(k) \otimes \mathbb{Q}$.

It is likely that considering the extension $\mathcal{B}(k[\varepsilon]$ ) (see Section 2) of the group $\mathcal{B}(k)$ to the ring $k[\varepsilon]$ of dual numbers, these exact sequences could be generalized 
as sequences (note that the "weight 1 "part in $\varepsilon$ of the first one was investigated in [6])

$$
0 \rightarrow \mu(k) \rightarrow H_{3}\left(\mathrm{SL}_{2}(k[\varepsilon]), \mathbb{Z}\right) \rightarrow \mathcal{B}(k[\varepsilon]) \rightarrow \bigwedge^{2} k[\varepsilon]^{\times} \rightarrow H_{2}\left(\mathrm{SL}_{2}(k[\varepsilon]), \mathbb{Z}\right) \rightarrow 0
$$

and

$$
0 \rightarrow K_{3}^{(2)}(k[\varepsilon]) \rightarrow \mathcal{B}(k[\varepsilon]) \otimes \mathbb{Q} \rightarrow \bigwedge^{2} k[\varepsilon]^{\times} \rightarrow K_{2}^{(2)}(k[\varepsilon]) \rightarrow 0 .
$$

For algebraically closed fields of characteristic 0 , the results of this paper could then be partially recovered, in the following way. Under the action of $k^{\times}$in $k[\varepsilon]$ : $a+\varepsilon b \mapsto a+\varepsilon t b$, we get weight decompositions of these exact sequences. More precisely, by calculations in cyclic homology [7], the weight decompositions of the $K$-groups involved reduce to

$$
\begin{aligned}
K_{3}^{(2)}(k[\varepsilon]) & =K_{3}^{(2)}(k) \oplus K_{3}^{(2)[3]}(k[\varepsilon]), \\
K_{2}^{(2)}(k[\varepsilon])=K_{2}(k[\varepsilon]) & =K_{2}^{(2)}(k) \oplus K_{2}^{(2)[1]}(k[\varepsilon]),
\end{aligned}
$$

where

$$
K_{3}^{(2)[3]}(k[\varepsilon]) \cong k, K_{2}^{(2)[1]}(k[\varepsilon]) \cong \Omega_{k}^{1} .
$$

This would imply the following exact sequences, which are in accordance with our results (in particular, the main result below is not a surprise)

$$
\begin{aligned}
0 \rightarrow k & \rightarrow \mathcal{B}(k[\varepsilon])^{[3]} \rightarrow 0 \\
0 & \rightarrow \mathcal{B}(k[\varepsilon])^{[2]} \rightarrow \bigwedge^{2} k \rightarrow 0 \\
0 & \rightarrow \mathcal{B}(k[\varepsilon])^{[1]} \rightarrow k \otimes k^{\times} \rightarrow \Omega_{k}^{1} \rightarrow 0 .
\end{aligned}
$$

Note that the two odd weight sequences are analogous to exact sequences appearing in the theory of scissors congruence, connected with the volume and the Dehn invariant (see [9]). The meaning of the one of weight two is not so clear (nevertheless see [19]).

In what follows, we consider the complex

$$
\begin{aligned}
\mathcal{B}(k[\varepsilon]) & \rightarrow \bigwedge^{2} k[\varepsilon]^{\times} \\
u & \mapsto u \wedge(1-u),
\end{aligned}
$$

for any field. One main result connected to the study of this complex is the following.

TheOREM 1.1. - Let $k$ be a field. If $\operatorname{Char}(k) \neq 2,3, \mathcal{B}(k[\varepsilon])$ is isomorphic as an abelian group to

$$
\mathcal{B}(k) \bigoplus \beta(k) \bigoplus \bigwedge^{2} k \bigoplus k
$$

where the space $\beta(k)$ was introduced in [6] (see Section 8). 
In general, there is a filtration $F_{0}=\mathcal{B}(k[\varepsilon]) \supset F_{1} \supset F_{2} \supset F_{3}$, such that $\mathcal{B}(k[\varepsilon])=\mathcal{B}(k) \oplus F_{1}$ and

1) $F_{3} \cong k$, if $\operatorname{Char}(k) \neq 2,3$.

2) $F_{2} / F_{3} \cong \bigwedge^{2} k$, if $\operatorname{Char}(k) \neq 2$.

3) $F_{1} / F_{2} \cong \beta(k)$, without any hypothesis on $k$.

For another main result, see Theorem 8.13.

In an appendix we give partial results on the extension to the dual numbers of the trilogarithmic complex of Goncharov.

To conclude this introduction, it is also likely that the fine results of Suslin in [31], which address all fields, can be extended to dual numbers. The results of this paper could then be applied to $K_{3}^{\text {ind }}(k[\varepsilon])$, for general fields.

\section{The Bloch-Suslin complex of a commutative ring}

For $A$ a commutative ring, let $A^{\times}$be the multiplicative group of invertible elements of $A$. We define $\mathcal{B}(A)$ as the commutative group with generators symbols $\{\alpha\}$, such that $\alpha, 1-\alpha \in A^{\times}$, submitted to the five terms relation

$$
\{\alpha\}-\left\{\alpha^{\prime}\right\}+\left\{\frac{\alpha^{\prime}}{\alpha}\right\}-\left\{\frac{1-\alpha^{\prime}}{1-\alpha}\right\}+\left\{\frac{\alpha\left(1-\alpha^{\prime}\right)}{\alpha^{\prime}(1-\alpha)}\right\}=0
$$

where all the elements: $\alpha, 1-\alpha, \alpha^{\prime}, 1-\alpha^{\prime}$ and $\alpha^{\prime}-\alpha$ are in $A^{\times}$. We can then consider the complex

$$
\mathcal{B}(A) \stackrel{D}{\rightarrow} \bigwedge^{2} A^{\times}
$$

with

$$
D(\{\alpha\})=\alpha \wedge(1-\alpha) .
$$

This extension of the Bloch-Suslin complex is probably reasonable only for commutative rings with many units.

Let $\mathcal{G}(A)$ (resp. $\mathcal{R}(A)$ ) be the free $\mathbb{Z}$-module generated by symbols $|\alpha|$, with $\alpha, 1-\alpha \in A^{\times}$(resp. symbols $\left|\alpha, \alpha^{\prime}\right|$, with $\alpha, 1-\alpha, \alpha^{\prime}, 1-\alpha^{\prime}, \alpha^{\prime}-\alpha \in A^{\times}$). For further purpose, we note the exact sequence

$$
\begin{gathered}
\mathcal{R}(A) \rightarrow \mathcal{G}(A) \rightarrow \mathcal{B}(A) \rightarrow 0 \\
|\alpha| \mapsto\{\alpha\} \\
\left|\alpha, \alpha^{\prime}\right| \mapsto\left|\alpha^{\prime}\right|-\left|\alpha^{\prime}\right|+\left|\frac{\alpha^{\prime}}{\alpha}\right|-\left|\frac{1-\alpha^{\prime}}{1-\alpha}\right|+\left|\frac{\alpha\left(1-\alpha^{\prime}\right)}{\alpha^{\prime}(1-\alpha)}\right| .
\end{gathered}
$$

TOME $135-2007-\mathrm{N}^{\mathrm{O}} 4$ 


\section{The tangent complex to the Bloch-Suslin complex of a field}

Let $k$ be an arbitrary field, $k_{0}$ its prime field and $k[\varepsilon]$ be the ring of dual numbers. For any functor $\mathcal{F}$ from commutative rings to abelian groups, the action of $k^{\times}$in $k[\varepsilon]: a+\varepsilon b \mapsto a+\varepsilon t b$, induces an action $u \mapsto t \star u$ of $k^{\times}$on $\mathcal{F}(k[\varepsilon])$. We denote by $\pi$ the map: $\mathcal{F}(k[\varepsilon]) \rightarrow \mathcal{F}(k)$, induced by the projection: $k[\varepsilon] \rightarrow k, \quad \varepsilon \mapsto 0$, and define $T \mathcal{F}(k)$ as the kernel of $\pi$.

Lemma 3.1. - The tangent group $T \mathcal{B}(k)$ can be presented, as a $\mathbb{Z}$-module, by generators

$$
\langle a ; b], a, b \in k, a \neq 0,1,
$$

where $\langle a ; b]=\{a+\varepsilon b\}-\{a\}$, with defining relations

$$
\begin{aligned}
\langle a ; b]-\left\langle a^{\prime} ;\right. & \left.b^{\prime}\right]+\left\langle\frac{a^{\prime}}{a} ; \frac{1}{a^{2}}\left(a b^{\prime}-a^{\prime} b\right)\right] \\
& -\left\langle\frac{1-a^{\prime}}{1-a} ; \frac{1}{(1-a)^{2}}\left(\left(1-a^{\prime}\right) b-(1-a) b^{\prime}\right)\right] \\
& +\left\langle\frac{a\left(1-a^{\prime}\right)}{a^{\prime}(1-a)} ; \frac{1}{\left(a^{\prime}(1-a)\right)^{2}}\left(a^{\prime}\left(1-a^{\prime}\right) b-a(1-a) b^{\prime}\right)\right]=0,
\end{aligned}
$$

for $a, a^{\prime} \neq 0,1, a \neq a^{\prime}$.

Proof. - The exact sequence of Section 2

$$
\mathcal{R}(k[\varepsilon]) \rightarrow \mathcal{G}(k[\varepsilon]) \rightarrow \mathcal{B}(k[\varepsilon]) \rightarrow 0,
$$

splits as the direct sum of the following two other ones

$$
\begin{gathered}
\mathcal{R}(k) \rightarrow \mathcal{G}(k) \rightarrow \mathcal{B}(k) \rightarrow 0 \\
T \mathcal{R}(k) \rightarrow T \mathcal{G}(k) \rightarrow T \mathcal{B}(k) \rightarrow 0 .
\end{gathered}
$$

We remark that $T \mathcal{G}(k)$ (resp. $T \mathcal{R}(k)$ ) is the free $\mathbb{Z}$-module with basis the elements $w-\pi(w)$, associated to the generators $w$ of $\mathcal{G}(k[\varepsilon])$ (resp. $T \mathcal{R}(k[\varepsilon]))$. As a result, by simple calculations in $k[\varepsilon]$, we get the defining relations of the lemma.

We note the canonical isomorphism

$$
\begin{gathered}
\bigwedge^{2} k[\varepsilon]^{\times} \rightarrow \bigwedge^{2} k^{\times} \bigoplus\left(k \otimes k^{\times}\right) \bigoplus \bigwedge^{2} k \\
(a+\varepsilon b) \wedge\left(a^{\prime}+\varepsilon b^{\prime}\right) \mapsto a \wedge a^{\prime} \oplus\left(a \otimes \frac{b^{\prime}}{a^{\prime}}-a^{\prime} \otimes \frac{b}{a}\right) \oplus \frac{b}{a} \wedge \frac{b^{\prime}}{a^{\prime}} .
\end{gathered}
$$

The purpose of what follows is to clarify the cohomological complex placed in degree 1 and 2

$$
D: T \mathcal{B}(k) \rightarrow\left(k \otimes k^{\times}\right) \times \wedge^{2} k
$$




$$
D(\langle a ; b])=\left(\frac{b}{a} \otimes(1-a)+\frac{b}{1-a} \otimes a,-\frac{b}{a} \wedge \frac{b}{1-a}\right) .
$$

\section{The filtration of $T \mathcal{B}(k)$}

Recall that $\langle a ; b]=\{a+\varepsilon b\}-\{a\}$. For $n \geq 2, a \in k \backslash\{0,1\}$ and $b_{i} \in k$, we define by induction the following elements in $T \mathcal{B}(k)$

$$
\begin{aligned}
& \left\langle a ; b_{1}, \ldots, b_{n}\right]= \\
& \qquad \begin{aligned}
&\left\langle a ; b_{1}, \ldots, b_{n-2}, b_{n-1}+b_{n}\right]-\left\langle a ; b_{1}, \ldots, b_{n-2}, b_{n-1}\right] \\
&-\left\langle a ; b_{1}, \ldots, b_{n-2}, b_{n}\right] .
\end{aligned}
\end{aligned}
$$

Note that for any permutation $\sigma$

$$
\left\langle a ; b_{\sigma(1)}, \ldots, b_{\sigma(n)}\right]=\left\langle a ; b_{1}, \ldots, b_{n}\right],
$$

and for $1 \leq i \leq n$

$$
\left\langle a ; b_{1}, \ldots, b_{i-1}, 0, b_{i+1}, \ldots, b_{n}\right]=0 .
$$

We get a decreasing filtration: $F_{1}=T \mathcal{B}(k) \supset F_{2} \supset F_{3} \supset \cdots \supset F_{n} \supset \ldots$, where $F_{n}$ is the subgroup generated by the elements $\left\langle a ; b_{1}, \ldots, b_{n}\right]$. The $\star$-action of $k^{\times}$on $\mathcal{B}(k[\varepsilon])$ respects this filtration.

Let $\left\langle a ; b_{i}, i=1, \ldots, n\right]$ denote $\left\langle a ; b_{1}, \ldots, b_{n}\right]$, for $n \geq 1$ we get from (1) the 5 -term relations

$$
\begin{aligned}
& \left\langle a ; b_{i}, i=1, \ldots, n\right]-\left\langle a^{\prime} ; b_{i}^{\prime}, i=1, \ldots, n\right] \\
& +\left\langle\frac{a^{\prime}}{a} ; \frac{1}{a^{2}}\left(a b_{i}^{\prime}-a^{\prime} b_{i}\right), i=1, \ldots, n\right] \\
& \quad-\left\langle\frac{1-a^{\prime}}{1-a} ; \frac{1}{(1-a)^{2}}\left(\left(1-a^{\prime}\right) b_{i}-(1-a) b_{i}^{\prime}\right), i=1, \ldots, n\right] \\
& +\left\langle\frac{a\left(1-a^{\prime}\right)}{a^{\prime}(1-a)} ; \frac{1}{\left(a^{\prime}(1-a)\right)^{2}}\left(a^{\prime}\left(1-a^{\prime}\right) b_{i}-a(1-a) b_{i}^{\prime}\right), i=1, \ldots, n\right]=0 .
\end{aligned}
$$

We collect the following simple facts: recall that $k_{0}$ is the prime field of $k$.

\section{Proposition 4.1. - We have}

(i) $F_{4}=0$, and then the symbol $\left\langle a ; b_{1}, b_{2}, b_{3}\right]$ is multiadditive.

(ii) $F_{1} / F_{2} \oplus F_{2} / F_{3} \oplus F_{3}$ is a $k_{0}$-vector space.

(iii) The $\star$-action induces a structure of $k$-vector space on $F_{1} / F_{2}$.

(iv) Let $n=1,2,3$. 
For $k$ of characteristic $0, F_{n}$ is a $\mathbb{Q}$-vector space.

For $k$ of characteristic $p, F_{n}$ is a $\frac{\mathbb{Z}}{p^{(4-n)} \mathbb{Z}}$-module.

In particular:

If $\operatorname{Char}(k) \neq 2, F_{n}$ is a $\mathbb{Z}[1 / 2]$-module.

If $\operatorname{Char}(k) \neq 2,3, F_{n}$ is a $\mathbb{Z}[1 / 6]$-module.

Proof. - The assertion i) stems from the choices

$$
b_{1}^{\prime}=0, b_{2}^{\prime}=\frac{a^{\prime}}{a} b_{1}, b_{3}^{\prime}=\frac{1-a^{\prime}}{1-a} b_{2}, b_{4}^{\prime}=\frac{a^{\prime}\left(1-a^{\prime}\right)}{a(1-a)} b_{3},
$$

in (2), with $n=4$.

We pass to ii). Let $\left\langle\left\langle a ; b_{1}, \ldots, b_{n}\right]\right.$ be the class of $\left\langle a ; b_{1}, \ldots, b_{n}\right]$ in $F_{n} / F_{n+1}$, for $n=1,2$. When $k_{0}=\mathbb{F}_{p}, F_{n} / F_{n+1}$ is $p$-torsion, since from the multiadditivity of the symbol $\left\langle\left\langle a ; b_{1}, \ldots, b_{n}\right]\right.$

$$
p\left\langle\left\langle a ; b_{1}, b_{2}, \ldots, b_{n}\right]=\left\langle\left\langle a ; p b_{1}, b_{2}, \ldots, b_{n}\right]=0 .\right.\right.
$$

If $k$ is of characteristic 0 , we have to prove that $F_{n} / F_{n+1}$ is uniquely divisible. This uses the induced $\star$-action of $k^{\times}$in $F_{n} / F_{n+1}$. By the multiadditivity of $\left\langle\left\langle a ; b_{1}, \ldots, b_{n}\right] \in F_{n} / F_{n+1}\right.$, we have $q \star v=q^{n} v$, for $v \in F_{n} / F_{n+1}$ and $q$ an integer; therefore $v=q u$, with $u=q^{n-1}\left(q^{-1} \star v\right)$, and if $q v=q w$, $v=q^{-1} \star(q \star v)=q^{-1} \star q^{n} v=q^{-1} \star q^{n} w=w$.

The point iii) is clear.

For iv) we just remark that an extension of uniquely divisible abelian groups is uniquely divisible.

Corollary 4.2. - If $\operatorname{Char}(k) \neq 2,3$, the exact sequence

$$
0 \rightarrow F_{3} \rightarrow F_{1} \rightarrow F_{1} / F_{3} \rightarrow 0,
$$

splits.

If $\operatorname{Char}(k) \neq 2$, the exact sequence

$$
0 \rightarrow F_{2} / F_{3} \rightarrow F_{1} / F_{3} \rightarrow F_{1} / F_{2} \rightarrow 0,
$$

splits.

Proof. - The relations (2) imply that there is a well defined morphism

$$
T \mathcal{B}(k) \rightarrow F_{n},
$$

such that

$$
\langle a ; b] \mapsto\langle a ; \underbrace{b, \ldots, b}_{n}] .
$$

Thus, If $\operatorname{Char}(k) \neq 2,3$, we get a morphism of abelian groups $F_{1} \rightarrow F_{3}$ such that $\langle a ; b] \mapsto \frac{1}{6}\langle a ; b, b, b]$. It is a retraction of the inclusion $F_{3} \rightarrow F_{1}$. 
In the same way, if $\operatorname{Char}(k) \neq 2$, we get a morphism $F_{1} \rightarrow F_{2}$, such that $\langle a ; b] \mapsto \frac{1}{2}\langle a ; b, b]$. The induced morphism $F_{1} / F_{3} \rightarrow F_{2} / F_{3}$ is a retraction of the inclusion $F_{2} / F_{3} \rightarrow F_{1} / F_{3}$.

We write $D_{1 / 2}, D_{2 / 3}$, the natural maps induced by $D$

$$
\begin{gathered}
D_{1 / 2}: F_{1} / F_{2} \rightarrow k \otimes k^{\times} \\
\left\langle\langle a ; b] \mapsto \frac{b}{a} \otimes(1-a)+\frac{b}{1-a} \otimes a,\right.
\end{gathered}
$$

and

$$
\begin{gathered}
D_{2 / 3}: F_{2} / F_{3} \rightarrow \bigwedge^{2} k \\
\left\langle\left\langle a ; b_{1}, b_{2}\right] \mapsto \frac{b_{1}}{1-a} \wedge \frac{b_{2}}{a}-\frac{b_{1}}{a} \wedge \frac{b_{2}}{1-a} .\right.
\end{gathered}
$$

REMARK 4.3. - Assume that the characteristic of $k$ is $\neq 2,3$, then by the previous corollary, $T \mathcal{B}(k)=F_{1}$ is a $k_{0}$-vector space. Let $\Lambda$ be the automorphism $T \mathcal{B}(k)=F_{1}$, given by the $\star$-action by 2 . Then, by elementary linear algebra, $\Lambda$ is diagonalizable with eigenvalues $2,2^{2}, 2^{3}$, and $F_{3}$ is exactly the eigenspace for the eigenvalue $2^{3}$. Also if $F_{1}=F_{1}^{\prime} \oplus F_{2}^{\prime} \oplus F_{3}$ is the diagonalization, then $F_{2}=F_{2}^{\prime} \oplus F_{3}$, and the natural map $F_{1}^{\prime} \rightarrow F_{1} / F_{2}$ (resp. $\left.F_{2}^{\prime} \rightarrow F_{2} / F_{3}\right)$ is an isomorphism whose inverse is induced by the projection $1 / 12\left(\Lambda^{2}-12 \Lambda+32 I d\right)$ (resp. $1 / 2(\Lambda-8 I d)$ ). In particular in this case, the tangent complex splits as the direct sum of the three complexes

$$
F_{1}^{\prime} \rightarrow k \otimes k^{\times}, \quad F_{2}^{\prime} \rightarrow \bigwedge^{2} k, \quad F_{3} \rightarrow 0 .
$$

Note that the tangent group to the Bloch group, considered by Bloch-Esnault in [3], corresponds to $F_{1}^{\prime} \oplus F_{3}$.

\section{A few relations on symbols}

In this section we collect a series of identities on symbols.

LEMmA 5.1. - We have the following relations in $\mathrm{TB}(k)$, where $a, a^{\prime} \neq$ $0,1, a \neq a^{\prime}$,

$$
\begin{aligned}
\langle a ; a(1-a) b]-\left\langle a^{\prime} ;\right. & \left.a^{\prime}\left(1-a^{\prime}\right) b\right]+\left\langle\frac{a^{\prime}}{a} ; \frac{a^{\prime}}{a}\left(1-\frac{a^{\prime}}{a}\right) a b\right] \\
& -\left\langle\frac{1-a^{\prime}}{1-a} ;-\frac{1-a^{\prime}}{1-a}\left(1-\frac{1-a^{\prime}}{1-a}\right)(1-a) b\right]=0 .
\end{aligned}
$$

By the $\star$-action, this relation is equivalent to

$$
\langle a\rangle-\left\langle a^{\prime}\right\rangle+a \star\left\langle\frac{a^{\prime}}{a}\right\rangle-(a-1) \star\left\langle\frac{1-a^{\prime}}{1-a}\right\rangle=0,
$$


where $\langle a\rangle:=\langle a ; a(1-a)]$.

$$
\begin{aligned}
\left\langle a ;(1-a) b_{1}, a(1-a) b_{2}\right] & -\left\langle a^{\prime} ;\left(1-a^{\prime}\right) b_{1}, a^{\prime}\left(1-a^{\prime}\right) b_{2}\right] \\
+ & \left\langle\frac{a^{\prime}}{a} ;\left(1-\frac{a^{\prime}}{a}\right) a^{-1} b_{1}, \frac{a^{\prime}}{a}\left(1-\frac{a^{\prime}}{a}\right) a b_{2}\right]=0 .
\end{aligned}
$$

$$
\left\langle a ; a b_{1},(1-a) b_{2}, a(1-a) b_{3}\right]=\left\langle a^{\prime} ; a^{\prime} b_{1},\left(1-a^{\prime}\right) b_{2}, a^{\prime}\left(1-a^{\prime}\right) b_{3}\right] .
$$

Proof. — Taking respectively in (2)

$$
\begin{array}{ll}
- \text { for } n=1 & b^{\prime}=\frac{a^{\prime}\left(1-a^{\prime}\right)}{a(1-a)} b, \\
- \text { for } n=2 & b_{1}^{\prime}=\frac{1-a^{\prime}}{1-a} b_{1}, b_{2}^{\prime}=\frac{a^{\prime}\left(1-a^{\prime}\right)}{a(1-a)} b_{2}, \\
- \text { for } n=3 & b_{1}^{\prime}=\frac{a^{\prime}}{a} b_{1}, b_{2}^{\prime}=\frac{1-a^{\prime}}{1-a} b_{2}, b_{3}^{\prime}=\frac{a^{\prime}\left(1-a^{\prime}\right)}{a(1-a)} b_{3},
\end{array}
$$

we get the following 4, 3 and 2-term relations

$$
\begin{aligned}
\langle a ; b]- & \left\langle a^{\prime} ; \frac{a^{\prime}\left(1-a^{\prime}\right)}{a(1-a)} b\right]+\left\langle\frac{a^{\prime}}{a} ; \frac{a^{\prime}\left(a-a^{\prime}\right)}{a^{2}(1-a)} b\right] \\
- & \left\langle\frac{1-a^{\prime}}{1-a} ; \frac{\left(1-a^{\prime}\right)\left(a-a^{\prime}\right)}{a(1-a)^{2}} b\right]=0, \\
\left\langle a ; b_{1}, b_{2}\right]- & \left\langle a^{\prime} ; \frac{1-a^{\prime}}{1-a} b_{1}, \frac{a^{\prime}\left(1-a^{\prime}\right)}{a(1-a)} b_{2}\right] \\
+ & \left\langle\frac{a^{\prime}}{a} ; \frac{a-a^{\prime}}{a^{2}(1-a)} b_{1}, \frac{a^{\prime}\left(a-a^{\prime}\right)}{a^{2}(1-a)} b_{2}\right]=0,
\end{aligned}
$$

and $\quad\left\langle a ; b_{1}, b_{2}, b_{3}\right]=\left\langle a^{\prime} ; \frac{a^{\prime}}{a} b_{1}, \frac{1-a^{\prime}}{1-a} b_{2}, \frac{a^{\prime}\left(1-a^{\prime}\right)}{a(1-a)} b_{3}\right]$.

The relations of the lemma follow.

LEMmA 5.2. - If $\operatorname{Char}(k) \neq 2$, we have the following relations in $T \mathcal{B}(k)$, for $a \neq 0,1$,

$$
\langle a\rangle+(-1) \star\langle 1-a\rangle=0,
$$

or, what is equivalent, using the $\star$-action $\langle a ; b]=-\langle 1-a ;-b]$,

$$
\begin{gathered}
\left\langle a ; b_{1}, b_{2}\right]=-\left\langle 1-a ;-b_{1},-b_{2}\right] \\
\left\langle a ; b_{1}, b_{2}, b_{3}\right]=\left\langle 1-a ; b_{1}, b_{2}, b_{3}\right] .
\end{gathered}
$$


Proof. - Changing in (4): $a$ in $1-a, a^{\prime}$ in $1-a^{\prime}$, and using the $\star$-action by -1 , we get

$$
(-1) \star\langle 1-a\rangle-(-1) \star\left\langle 1-a^{\prime}\right\rangle+(a-1) \star\left\langle\frac{1-a^{\prime}}{1-a}\right\rangle-a \star\left\langle\frac{a^{\prime}}{a}\right\rangle=0 .
$$

By difference with (4), we see that $\langle a\rangle+(-1) \star\langle 1-a\rangle$ is a constant, but permuting $a$ and $1-a$, this shows that $2(\langle a\rangle+(-1) \star\langle 1-a\rangle)=0$. By 2divisibility this gives $(7)$. The other relations follow: note the multiadditivity of the symbol $\left\langle a ; b_{1}, b_{2}, b_{3}\right]$ in $F_{3}$ which is responsible for the sign in iii).

Lemma 5.3. - If Char $(k) \neq 2$, we have the following relations, with $a, a^{\prime} \neq$ 0,1 and $a \neq a^{\prime}$,

(i) In $F_{1} / F_{2}$, where $[a]$ is the class of $\langle a\rangle$

$$
\begin{gathered}
{[a]-\left[a^{\prime}\right]+a\left[\frac{a^{\prime}}{a}\right]+(1-a)\left[\frac{1-a^{\prime}}{1-a}\right]=0 .} \\
{[a]-[1-a]=0 .} \\
{[a]+a\left[\frac{1}{a}\right]=0 .}
\end{gathered}
$$

By the $\star$-action, this last relation is equivalent to

$$
\left\langle\langle a ; b]=\left\langle\left\langle\frac{1}{a} ; \frac{b}{a^{2}}\right] .\right.\right.
$$

(ii) In $F_{2} / F_{3}$,

(iii) In $F_{3}$,

$$
\left\langle\left\langle a ; b_{1}, b_{2}\right]=-\left\langle\left\langle\frac{1}{a} ; \frac{b_{1}}{a^{2}}, \frac{b_{2}}{a^{2}}\right] .\right.\right.
$$

$$
\left\langle a ; b_{1}, b_{2}, b_{3}\right]=\left\langle\frac{1}{a} ; \frac{b_{1}}{a^{2}}, \frac{b_{2}}{a^{2}}, \frac{b_{3}}{a^{2}}\right] .
$$

Proof. - We remark that for $w$ in $F_{1} / F_{2}, F_{2} / F_{3}$ or $F_{3},(-1) \star w=-w$.

In $F_{1} / F_{2}$, the relation (4) combined with (7) writes

$$
[a]-\left[a^{\prime}\right]+a\left[\frac{a^{\prime}}{a}\right]+(1-a)\left[\frac{1-a^{\prime}}{1-a}\right]=0,
$$

and we have

$$
[a]-[1-a]=0 \text {. }
$$

Taking $a^{\prime}=1-a$, we get

$$
a\left[\frac{1-a}{a}\right]+(1-a)\left[\frac{a}{1-a}\right]=0
$$

which implies (10) if $a \neq-1$, the case $a=-1$ being trivial.

The proofs of ii) and iii) are similar, but we have to start directly from (2), taken for $n=2$ or 3 . 
An easy refinement of the proofs of the previous lemmas gives

LEMMA 5.4. - In any characteristic the following relation, for $a, a^{\prime} \neq 0,1$ and $a \neq a^{\prime}$, is satisfied in $F_{1} / F_{2}$

$$
[a]-\left[a^{\prime}\right]+a\left[\frac{a^{\prime}}{a}\right]+(1-a)\left[\frac{1-a^{\prime}}{1-a}\right]=0 .
$$

It implies, for $a \neq 0,1$, the relations

$$
\begin{gathered}
{[a]-[1-a]=\omega,} \\
{[a]+a\left[\frac{1}{a}\right]+(1-a) \omega=0,}
\end{gathered}
$$

where $\omega \in F_{1} / F_{2}$ does not depend on $a$ and $2 \omega=0$.

\section{Calculation of $F_{3}$ : the "additive dilogarithm"}

In this section we determine the weight 3 piece $F_{3}$, under the hypothesis that $\operatorname{Char}(k) \neq 2,3$. We first remark the following

Proposition 6.1. - There is a morphism of abelian groups (which might be called the additive dilogarithm)

$$
\mathcal{L}^{+}: T \mathcal{B}(k) \rightarrow k
$$

such that

$$
\langle a ; b] \mapsto \frac{b^{3}}{6(a(1-a))^{2}}
$$

Proof. - For the existence of this morphism, we use the presentation of $T \mathcal{B}(k)$ from Section 3

$$
T \mathcal{R}(k) \rightarrow T \mathcal{G}(k) \rightarrow T \mathcal{B}(k) \rightarrow 0
$$

The assignment

$$
\langle a ; b] \mapsto \frac{b^{3}}{6(a(1-a))^{2}}
$$

is compatible with the 5 -term relations (1): to see this, one has to prove the following identity in $k$

$$
\begin{gathered}
\frac{b^{3}}{(a(1-a))^{2}}-\frac{b^{\prime 3}}{\left(a^{\prime}\left(1-a^{\prime}\right)\right)^{2}}+\frac{\left(a b^{\prime}-a^{\prime} b\right)^{3}}{\left(a a^{\prime}\left(a-a^{\prime}\right)\right)^{2}} \\
-\frac{\left(\left(1-a^{\prime}\right) b-(1-a) b^{\prime}\right)^{3}}{\left((1-a)\left(1-a^{\prime}\right)\left(a-a^{\prime}\right)\right)^{2}}+\frac{\left(a^{\prime}\left(1-a^{\prime}\right) b-a(1-a) b^{\prime}\right)^{3}}{\left(a a^{\prime}(1-a)\left(1-a^{\prime}\right)\left(a-a^{\prime}\right)\right)^{2}}=0 .
\end{gathered}
$$

But considering this expression as a polynomial in $b, b^{\prime}$, the coefficients are easily checked to be 0 . 
THEOREM 6.2. - There is an isomorphism of $k_{0}$-vector spaces

$$
\mathcal{L}: F_{3} \cong k \text {. }
$$

such that for $a \neq 0,1$

$$
\mathcal{L}\left(\left\langle a ; b_{1}, b_{2}, b_{3}\right]\right)=\frac{b_{1} b_{2} b_{3}}{(a(1-a))^{2}} .
$$

Proof. - Let $\mathcal{L}$ be the restriction of $\mathcal{L}^{+}$to $F_{3}$. The equality

$\left\langle a ; b_{1}, b_{2}, b_{3}\right]=\left\langle a ; b_{1}+b_{2}+b_{3}\right]-\left\langle a ; b_{1}+b_{2}\right]-\left\langle a ; b_{1}+b_{3}\right]-\left\langle a ; b_{2}+b_{3}\right]$

$$
+\left\langle a ; b_{1}\right]+\left\langle a ; b_{2}\right]+\left\langle a ; b_{3}\right]
$$

implies

$$
\mathcal{L}\left(\left\langle a ; b_{1}, b_{2}, b_{3}\right]\right)=\frac{b_{1} b_{2} b_{3}}{(a(1-a))^{2}}
$$

We construct now the inverse of $\mathcal{L}$. From (6) the symbol

$$
\left\langle a ; a b_{1},(1-a) b_{2}, a(1-a) b_{3}\right],
$$

does not depend on $a \neq 0,1$. Let us define new multiadditive symbols

$$
b_{1} \bullet b_{2} \bullet b_{3}:=\left\langle a ; a b_{1},(1-a) b_{2}, a(1-a) b_{3}\right] .
$$

Since

$$
\left\langle a ; b_{1}, b_{2}, b_{3}\right]=\frac{b_{1}}{a} \bullet \frac{b_{2}}{1-a} \bullet \frac{b_{3}}{a(1-a)}
$$

these symbols span $F_{3}$.

Lemma 6.3. - We have the relation

$$
b_{1} \bullet b_{2} \bullet b_{3}=b_{1} b_{2} b_{3} \bullet 1 \bullet 1 \text {. }
$$

Proof. - From the symmetry of the symbol $\left\langle a ; b_{1}, b_{2}, b_{3}\right]$, we derive the relations

$$
b_{1} \bullet b_{2} \bullet b_{3}=\frac{1-a}{a} b_{2} \bullet \frac{a}{1-a} b_{1} \bullet b_{3},
$$

and

$$
b_{1} \bullet b_{2} \bullet b_{3}=(1-a) b_{3} \bullet b_{2} \bullet \frac{1}{1-a} b_{1} \text {. }
$$

Since $\left(-b_{1}\right) \bullet b_{2} \bullet b_{3}=-b_{1} \bullet b_{2} \bullet b_{3}$, we can assume that $b_{1} \neq 0,-1$. Letting either $a=\frac{1}{1+b_{1}}$ or $a=1+b_{1}$, in the previous relations, gives the equalities

$$
b_{1} \bullet b_{2} \bullet b_{3}=b_{1} b_{2} \bullet 1 \bullet b_{3},
$$

and

$$
b_{1} \bullet b_{2} \bullet b_{3}=b_{1} b_{3} \bullet b_{2} \bullet 1 \text {. }
$$

This proves the lemma. 
From the lemma we get a surjective morphism of abelian groups

$$
\begin{aligned}
k & \rightarrow F_{3} \\
b & \mapsto b \bullet 1 \bullet 1 .
\end{aligned}
$$

This is the searched inverse.

REMARK 6.4. - The previous proof shows that $F_{3}$ is in fact the abelian group generated by symmetric and multiadditive symbols $\left\langle a ; b_{1}, b_{2}, b_{3}\right]$, submitted to the relations

$$
\left\langle a ; a b_{1},(1-a) b_{2}, a(1-a) b_{3}\right]=\left\langle a^{\prime} ; a^{\prime} b_{1},\left(1-a^{\prime}\right) b_{2}, a^{\prime}\left(1-a^{\prime}\right) b_{3}\right] .
$$

\section{Calculation of $F_{2} / F_{3}$}

We assume for this section that $\operatorname{Char}(k) \neq 2$. Recall that $\left\langle\left\langle a ; b_{1}, b_{2}\right]\right.$ denotes the class of $\left\langle a ; b_{1}, b_{2}\right]$ in $F_{2} / F_{3}$, and that $\left\langle\left\langle a ; b_{1}, b_{2}\right]\right.$ is biadditive.

THEOREM 7.1. - The morphism

$$
D_{2 / 3}: F_{2} / F_{3} \rightarrow \bigwedge^{2} k
$$

is bijective.

Proof. - We have for $a \neq 0,1$

$$
D_{2 / 3}\left(\left\langle a ; b_{1}, b_{2}\right]\right)=\frac{b_{1}}{1-a} \wedge \frac{b_{2}}{a}-\frac{b_{1}}{a} \wedge \frac{b_{2}}{1-a} .
$$

We remark that $D_{2 / 3}$ is onto. In fact the equality

$$
D_{2 / 3}(\langle\langle a ; a(1-a) b, a(1-a) b])=2 a b \wedge(1-a) b=2 a b \wedge b
$$

implies that for: $u, v, u+v \neq 0$,

$$
D_{2 / 3}\left(\frac{1}{2}\left\langle\left\langle\frac{u}{u+v} ; \frac{u v}{u+v}, \frac{u v}{u+v}\right]\right)=u \wedge v .\right.
$$

To built the inverse of $D_{2 / 3}$, we introduce the symbols

$$
u \odot v:=\left\langle\left\langle\frac{u}{u+v} ; \frac{u v}{u+v}, \frac{u v}{u+v}\right],\right.
$$

if $u, v$ and $u+v$ are $\neq 0$, and

$$
u \odot v:=0
$$

otherwise.

We have to prove that these symbols are antisymmetric and biadditive. The antisymmetry is an easy consequence of the relation

$$
\langle\langle a ; b, b]=-\langle\langle 1-a ; b, b] .
$$


The biadditivity of $u \odot v$ is less straightforward. We begin with a lemma.

LEMmA 7.2. - In presence of the $\star$-action, the biadditivity of $u \odot v$ is a consequence of the following relations

(i) For $a, a^{\prime}, a+a^{\prime} \neq 0,1$

$$
\begin{gathered}
\left\langle\left\langle a+a^{\prime} ;\left(a+a^{\prime}\right)\left(1-\left(a+a^{\prime}\right)\right),\left(a+a^{\prime}\right)\left(1-\left(a+a^{\prime}\right)\right)\right]=\right. \\
\left\langle\langle a ; a(1-a), a(1-a)]+\left\langle\left\langle a^{\prime} ; a^{\prime}\left(1-a^{\prime}\right), a^{\prime}\left(1-a^{\prime}\right)\right] .\right.\right.
\end{gathered}
$$

(ii) For $a \neq 0,1$

$$
\langle\langle a ; 1-a, 1-a]=-\langle\langle 1-a ; 1-a, 1-a]=\langle\langle 1+a ; 1+a, 1+a] .
$$

(iii) For $a \neq 0,1$

$$
\left\langle\langle a ; a, a]=-\left\langle\left\langle a^{-1} ; a^{-1}, a^{-1}\right] .\right.\right.
$$

Proof of the lemma. - Assume that $u, u^{\prime}, v, u+u^{\prime}, u+v, u^{\prime}+v, u+u^{\prime}+v$ are different from 0 . The relation

$$
\left(u+u^{\prime}\right) \odot v=u \odot v+u^{\prime} \odot v
$$

reads

$$
\begin{gathered}
\left\langle\left\langle\frac{u+u^{\prime}}{u+u^{\prime}+v} ; \frac{\left(u+u^{\prime}\right) v}{u+u^{\prime}+v}, \frac{\left(u+u^{\prime}\right) v}{u+u^{\prime}+v}\right]=\right. \\
\left.\ll \frac{u}{u+v} ; \frac{u v}{u+v}, \frac{u v}{u+v}\right]+《\left\langle\frac{u^{\prime}}{u^{\prime}+v} ; \frac{u^{\prime} v}{u^{\prime}+v}, \frac{u^{\prime} v}{u^{\prime}+v}\right] .
\end{gathered}
$$

But by the action of the multiplicative group $k^{\times}$, it is equivalent to the relation

$$
\begin{gathered}
\left.\ll \frac{u+u^{\prime}}{u+u^{\prime}+v} ; \frac{u+u^{\prime}}{u+u^{\prime}+v}, \frac{u+u^{\prime}}{u+u^{\prime}+v}\right]= \\
\left.\ll \frac{u}{u+v} ; \frac{u}{u+v}, \frac{u}{u+v}\right]+《\left\langle\frac{u^{\prime}}{u^{\prime}+v} ; \frac{u^{\prime}}{u^{\prime}+v}, \frac{u^{\prime}}{u^{\prime}+v}\right] .
\end{gathered}
$$

By the identity $\left\langle\langle a ; a, a]=-\left\langle\left\langle a^{-1} ; a^{-1}, a^{-1}\right]\right.\right.$, it is equivalent to

$$
\begin{gathered}
\left\langle\left\langle 1+\frac{v}{u+u^{\prime}} ; 1+\frac{v}{u+u^{\prime}}, 1+\frac{v}{u+u^{\prime}}\right]=\right. \\
\left\langle\ll 1+\frac{v}{u} ; 1+\frac{v}{u}, 1+\frac{v}{u}\right]+\left\langle\left\langle 1+\frac{v}{u^{\prime}} ; 1+\frac{v}{u^{\prime}}, 1+\frac{v}{u^{\prime}}\right] .\right.
\end{gathered}
$$

Let $a=-u / v$ and $a^{\prime}=-u^{\prime} / v$, we have $a, a^{\prime}, a+a^{\prime} \neq 0,1$. The previous relation becomes

$$
\begin{aligned}
\left\langle\left\langle 1-\frac{1}{a+a^{\prime}} ; 1-\right.\right. & \left.\frac{1}{a+a^{\prime}}, 1-\frac{1}{a+a^{\prime}}\right]= \\
& \quad\left\langle 1-\frac{1}{a} ; 1-\frac{1}{a}, 1-\frac{1}{a}\right]+\left\langle\left\langle 1-\frac{1}{a^{\prime}} ; 1-\frac{1}{a^{\prime}}, 1-\frac{1}{a^{\prime}}\right] .\right.
\end{aligned}
$$


Now applying the equality $\langle\langle 1-a ; b, b]=-\langle\langle a ; b, b]$, this is equivalent to

$$
\begin{aligned}
\left\langle\left\langle\frac{1}{a+a^{\prime}} ; 1-\frac{1}{a+a^{\prime}}, 1-\frac{1}{a+a^{\prime}}\right]=\right. \\
\quad\left\langle\left\langle\frac{1}{a} ; 1-\frac{1}{a}, 1-\frac{1}{a}\right]+\left\langle\left\langle\frac{1}{a^{\prime}} ; 1-\frac{1}{a^{\prime}}, 1-\frac{1}{a^{\prime}}\right] .\right.\right.
\end{aligned}
$$

And finally, from the relation $\left\langle\langle a ; b a, b a]=-\left\langle\left\langle a^{-1} ; b a^{-1}, b a^{-1}\right]\right.\right.$, we get the identity i) of the lemma.

The proof of ii) is similar and the relations there correspond to the cases where one of the terms $u+u^{\prime}, u+v, u^{\prime}+v, u+u^{\prime}+v$ is 0 , the other possibilities being trivial.

We now prove the relations of the lemma which are not already known. We first remark that by Lemma 5.3 ii)

$$
\left\langle\left\langle-1 ; b_{1}, b_{2}\right]=-\left\langle\left\langle-1 ; b_{1}, b_{2}\right] .\right.\right.
$$

Therefore by 2-divisibility

$$
\left\langle\left\langle-1 ; b_{1}, b_{2}\right]=0 .\right.
$$

Applying this to the three terms relation (5), with $a^{\prime}=-a$ and $b_{1}=b_{2}=$ $a(1-a)$, we obtain

$$
\langle\langle a ; a(1-a), a(1-a)]=-\langle\langle-a ; a(1+a), a(1+a)] .
$$

This implies

$$
\langle\langle 1-a ; a(1-a), a(1-a)]=-\langle\langle 1+a ; a(1+a), a(1+a)],
$$

which is equivalent by the $\star$-action to

$$
\langle\langle 1-a ; 1-a, 1-a]=-\langle\langle 1+a ; 1+a, 1+a] .
$$

We pass to the relation i) of the lemma. We can rewrite the 3 -terms relations (5) in the following form

$$
\begin{aligned}
& \quad\left\langle\left\langle\frac{a^{\prime}}{a} ; b_{1}, b_{2}\right]=\right. \\
& \quad\left\langle\left\langle a^{\prime} ; \frac{a^{2}\left(1-a^{\prime}\right)}{a-a^{\prime}} b_{1}, \frac{a\left(1-a^{\prime}\right)}{a-a^{\prime}} b_{2}\right]-\left\langle\left\langle a ; \frac{a^{2}(1-a)}{a-a^{\prime}} b_{1}, \frac{a^{2}(1-a)}{a^{\prime}\left(a-a^{\prime}\right)} b_{2}\right],\right.\right.
\end{aligned}
$$

which implies, changing $a$ in $1 / a$

$$
\begin{aligned}
& \left\langle\left\langle a a^{\prime} ; b_{1}, b_{2}\right]=\right. \\
& \quad\left\langle\left\langle a^{\prime} ; \frac{1-a^{\prime}}{a\left(1-a a^{\prime}\right)} b_{1}, \frac{1-a^{\prime}}{1-a a^{\prime}} b_{2}\right]+\left\langle\left\langle a ; \frac{1-a}{1-a a^{\prime}} b_{1}, \frac{1-a}{a^{\prime}\left(1-a a^{\prime}\right)} b_{2}\right] .\right.\right.
\end{aligned}
$$


Using these two identities, we have successively

$$
\begin{aligned}
\left\langle\left\langle a+a^{\prime} ; b_{1}, b_{2}\right]\right. & =\left\langle\left\langle a\left(1+\frac{a^{\prime}}{a}\right) ; b_{1}, b_{2}\right]\right. \\
& =\left\langle\left\langle 1+\frac{a^{\prime}}{a} ; \frac{a^{\prime}}{a^{2}\left(1-\left(a+a^{\prime}\right)\right)} b_{1}, \frac{a^{\prime}}{a\left(1-\left(a+a^{\prime}\right)\right)} b_{2}\right]\right. \\
& +\left\langle\left\langle a ; \frac{1-a}{1-\left(a+a^{\prime}\right)} b_{1}, \frac{a(1-a)}{\left(a+a^{\prime}\right)\left(1-\left(a+a^{\prime}\right)\right)} b_{2}\right]\right. \\
& =-\left\langle\left\langle-\frac{a^{\prime}}{a} ; \frac{a^{\prime}}{a^{2}\left(1-\left(a+a^{\prime}\right)\right)} b_{1}, \frac{a^{\prime}}{a\left(1-\left(a+a^{\prime}\right)\right)} b_{2}\right]\right. \\
& +\left\langle\left\langle a ; \frac{1-a}{1-\left(a+a^{\prime}\right)} b_{1}, \frac{a(1-a)}{\left(a+a^{\prime}\right)\left(1-\left(a+a^{\prime}\right)\right)} b_{2}\right]\right. \\
& =-\left\langle\left\langle-a^{\prime} ; \frac{a^{\prime}\left(1+a^{\prime}\right)}{\left(a+a^{\prime}\right)\left(1-\left(a+a^{\prime}\right)\right)} b_{1}, \frac{a^{\prime}\left(1+a^{\prime}\right)\left(1-\left(a+a^{\prime}\right)\right)}{\left(a+a_{2}\right)} b_{2}\right]\right. \\
& -\left\langle\left\langle a ; \frac{a^{\prime}(1-a)}{\left(a+a^{\prime}\right)\left(1-\left(a+a^{\prime}\right)\right)} b_{1}, \frac{a(1-a)}{\left(a+a^{\prime}\right)\left(1-\left(a+a^{\prime}\right)\right)} b_{2}\right]\right. \\
& +\left\langle\left\langle a ; \frac{1-a}{1-\left(a+a^{\prime}\right)} b_{1}, \frac{a(1-a)}{\left(a+a^{\prime}\right)\left(1-\left(a+a^{\prime}\right)\right)} b_{2}\right] .\right.
\end{aligned}
$$

Letting $b_{1}=b_{2}=\left(a+a^{\prime}\right)\left(1-\left(a+a^{\prime}\right)\right)$, we finally get by the biadditivity of the symbols and the relation (13)

$$
\begin{aligned}
& \left\langle\left\langle a+a^{\prime} ;\left(a+a^{\prime}\right)\left(1-\left(a+a^{\prime}\right),\left(a+a^{\prime}\right)\left(1-\left(a+a^{\prime}\right)\right]=\right.\right.\right. \\
& -\left\langle\left\langle-a^{\prime} ; a^{\prime}\left(1+a^{\prime}\right), a^{\prime}\left(1+a^{\prime}\right)\right]-\left\langle\left\langle a ; a^{\prime}(1-a), a(1-a)\right]\right.\right. \\
& \quad+\left\langle\left\langle a ;(1-a)\left(a+a^{\prime}\right), a(1-a)\right]\right. \\
& =\left\langle\langle a ; a(1-a), a(1-a)]+\left\langle\left\langle a^{\prime} ; a^{\prime}\left(1-a^{\prime}\right), a^{\prime}\left(1-a^{\prime}\right)\right] .\right.\right.
\end{aligned}
$$

We have thus proved the relations of the lemma. Now we can assert that there is a well defined morpism

$$
\bigwedge^{2} k \rightarrow F_{2} / F_{3}
$$

such that

$$
u \wedge v \mapsto \frac{1}{2}\left\langle\left\langle\frac{u}{u+v} ; \frac{u v}{u+v}, \frac{u v}{u+v}\right],\right.
$$

for $u, v$ and $u+v \neq 0$.

This is the left inverse to $D_{2 / 3}$, since $F_{2} / F_{3}$ is generated by the elements $\left\langle\langle a ; b, b]\right.$. Indeed we have in $F_{2} / F_{3}$

$$
\left\langle\left\langle a ; b_{1}, b_{2}\right]=\frac{1}{2}\left(\left\langle\left\langle a ; b_{1}+b_{2}, b_{1}+b_{2}\right]-\left\langle\left\langle a ; b_{1}, b_{1}\right]-\left\langle\left\langle a ; b_{2}, b_{2}\right]\right) .\right.\right.\right.\right.
$$

This achieves the proof of the theorem. 
REMARK 7.3. - For $a \neq 0$, we define in $F_{2} / F_{3}$

$$
\langle\langle a\rangle\rangle:=\langle\langle a ; a(1-a), a(1-a)] .
$$

Modulo the $\star$-action, the relations of Lemma 7.2 can be rewritten

$$
\begin{gathered}
\left\langle\left\langle a+a^{\prime}\right\rangle\right\rangle=\langle\langle a\rangle\rangle+\left\langle\left\langle a^{\prime}\right\rangle\right\rangle . \\
\langle\langle a\rangle\rangle+\langle\langle 1-a\rangle\rangle=0, \\
\langle\langle a\rangle\rangle+\langle\langle-a\rangle\rangle=0 . \\
\langle\langle a\rangle\rangle+a \star\left\langle\left\langle\frac{1}{a}\right\rangle\right\rangle=0 .
\end{gathered}
$$

The proofs of this section show incidentally that if $\operatorname{Char}(k) \neq 2, F_{2} / F_{3}$ is exactly the $\mathbb{Z}[1 / 2]\left[k^{\times}\right]$-module, generated by symbols $\langle\langle a\rangle\rangle$ for $a \neq 0,1$, submitted to the previous relations 1), 2) and 3). Another equivalent presentation is the following: $F_{2} / F_{3}$ is exactly the $\mathbb{Z}[1 / 2]\left[k^{\times}\right]$-module, generated by symbols $\langle\langle a\rangle\rangle$ for $a \in k$, submitted to the relations

$$
\begin{gathered}
\left\langle\left\langle a+a^{\prime}\right\rangle\right\rangle=\langle\langle a\rangle\rangle+\left\langle\left\langle a^{\prime}\right\rangle\right\rangle . \\
\langle\langle 0\rangle\rangle=\langle\langle 1\rangle\rangle=0 . \\
\langle\langle a\rangle\rangle+a \star\left\langle\left\langle\frac{1}{a}\right\rangle\right\rangle=0, \text { for } a \neq 0 .
\end{gathered}
$$

\section{The linearized tangent complex}

The linearized tangent complex to the Bloch-Suslin complex is the complex of $k$-vector spaces

$$
D_{1 / 2}: F_{1} / F_{2} \rightarrow k \otimes k^{\times} .
$$

We want to compute its cohomology. In this section the field $k$ is arbitrary. We complete the calculations in [6], in particular concerning the case of characteristic 2 which owes much to suggestions made to the author by the late Chi-Han Sah in 1986 [29].

8.1. Presentation of $F_{1} / F_{2}$. - We give in this section a simple presentation of $F_{1} / F_{2}$.

Definition 8.1. - The space $\beta(k)$ is defined as the $k$-linear space with generators symbols $[a], a \in k \backslash\{0,1\}$, and defining relations

$$
[a]-\left[a^{\prime}\right]+a\left[\frac{a^{\prime}}{a}\right]+(1-a)\left[\frac{1-a^{\prime}}{1-a}\right]=0 \text {, for } a, a^{\prime} \neq 0,1 \text { and } a \neq a^{\prime}
$$

Here is an avatar of $\beta(k)$. 
Definition 8.2. - For any field, $\beta^{\prime}(k)$ is the $k$-linear space generated by the symbols $[a]^{\prime}, a \in k$, with the defining relations,

$$
[1]^{\prime}=0,
$$

and

(16) $[a]^{\prime}-\left[a^{\prime}\right]^{\prime}+a\left[\frac{a^{\prime}}{a}\right]^{\prime}+(1-a)\left[\frac{1-a^{\prime}}{1-a}\right]^{\prime}=0$, for $a \neq 0,1$ and $a \neq a^{\prime}$.

Proposition 8.3. - The $k$-linear spaces $\beta(k)$ and $\beta^{\prime}(k)$ are canonically isomorphic.

Proof. - We have in $\beta^{\prime}(k)$ the following relations, for $a \neq 0,1$

$$
[a]^{\prime}-[1-a]^{\prime}=[0] \text {, }
$$

and

$$
[a]^{\prime}+a[1 / a]^{\prime}+(1-a)[0]^{\prime}=0 .
$$

For the second relation, take $a^{\prime}=1$ in (16). The first one is obtained by considering (16) with the data: either $a^{\prime}=1$ or $a^{\prime}=0$ with $1-a$ instead of $a$, and taking the difference.

On the other hand, by Lemma 5.4 , we have in $\beta(k)$ for $a \neq 0,1$

$$
[a]+a[1 / a]+(1-a) \omega=0,
$$

where $\omega$ does not depend on $a$ and is actually equal to $[a]-[1-a]$. This relation for $1-a$ instead of $a$ gives

$$
[1-a]+(1-a)\left[\frac{1}{1-a}\right]+a \omega=0
$$

which can be rewritten

$$
[a]-\omega+a \omega+(1-a)\left[\frac{1}{1-a}\right]=0 .
$$

All these relations imply that the correspondance $[a]^{\prime} \mapsto[a]$, for $a \neq 0,1$, $[0]^{\prime} \mapsto \omega$, induces an isomorphism from $\beta^{\prime}(k)$ to $\beta(k)$.

In what follows, the two spaces will be denoted the same way, and the presentation involved will be clear from the context.

Proposition 8.4. - Since the relations of definition of $\beta(k)$ are satisfied in $F_{1} / F_{2}$ by the results of Section 5 , we have a $k$-linear map, with $a \neq 0,1$

$$
\begin{aligned}
\beta(k) & \rightarrow F_{1} / F_{2} \\
{[a] } & \mapsto[a]=\langle\langle a ; a(1-a)] .
\end{aligned}
$$

This map is bijective.

TOME $135-2007-\mathrm{N}^{\mathrm{O}} 4$ 
Proof. - From (1), we know that the $k$-linear space $F_{1} / F_{2}$ is generated, as a commutative group, by symbols $\langle\langle a ; b]$, with defining relations

$$
\left\langle\left\langle a ; b_{1}+b_{2}\right]=\left\langle\left\langle a ; b_{1}\right]+\left\langle\left\langle a ; b_{2}\right],\right.\right.\right.
$$

and $\quad\left\langle\langle a ; b]-\left\langle\left\langle a^{\prime} ; b^{\prime}\right]+\left\langle\left\langle\frac{a^{\prime}}{a} ; \frac{1}{a^{2}}\left(a b^{\prime}-a^{\prime} b\right)\right]\right.\right.\right.$

$$
\begin{aligned}
& -\left\langle\left\langle\frac{1-a^{\prime}}{1-a} ; \frac{1}{(1-a)^{2}}\left(\left(1-a^{\prime}\right) b-(1-a) b^{\prime}\right)\right]\right. \\
& +\left\langle\left\langle\frac{a\left(1-a^{\prime}\right)}{a^{\prime}(1-a)} ; \frac{1}{\left(a^{\prime}(1-a)\right)^{2}}\left(a^{\prime}\left(1-a^{\prime}\right) b-a(1-a) b^{\prime}\right)\right]=0,\right.
\end{aligned}
$$

for $a, a^{\prime} \neq 0,1, a \neq a^{\prime}$.

Letting $b=0$ or $b^{\prime}=0$, the second relation can be replaced, in presence of the first, by the two relations

$$
\begin{aligned}
\langle\langle a ; b] & -\left\langle\left\langle\frac{a^{\prime}}{a} ; \frac{a^{\prime}}{a^{2}} b\right]-\left\langle\left\langle\frac{1-a^{\prime}}{1-a} ; \frac{1-a^{\prime}}{(1-a)^{2}} b\right]\right.\right. \\
+ & \left\langle\left\langle\frac{a\left(1-a^{\prime}\right)}{a^{\prime}(1-a)} ; \frac{1-a^{\prime}}{a^{\prime}(1-a)^{2}} b\right]=0,\right.
\end{aligned}
$$

and

$$
\begin{aligned}
\langle\langle a ; b] & -\left\langle\left\langle\frac{a}{a^{\prime}} ; \frac{b}{a^{\prime}}\right]-\left\langle\left\langle\frac{1-a}{1-a^{\prime}} ; \frac{b}{1-a^{\prime}}\right]\right.\right. \\
+ & \left\langle\left\langle\frac{a^{\prime}(1-a)}{a\left(1-a^{\prime}\right)} ; \frac{a^{\prime}}{\left(a^{2}\left(1-a^{\prime}\right)\right.} b\right]=0 .\right.
\end{aligned}
$$

As we can see, these two relations are in fact equivalent.

In characteristic $\neq 2$, this stems from the equality

$$
\left\langle\langle a ; b]=\left\langle\left\langle\frac{1}{a} ; \frac{b}{a^{2}}\right],\right.\right.
$$

applied to the three last terms of the first relation.

In characteristic 2 , the more complicated relation

$$
\left\langle\langle a ; b]=\frac{b}{a(1-a)}[a]=\left\langle\left\langle\frac{1}{a} ; \frac{b}{a^{2}}\right]+\frac{b}{a} \omega,\right.\right.
$$

is responsible for extra terms in $\omega$ which actually disappear, as one can check easily from the relation

$$
\frac{1}{a}+\frac{1}{1+a}+\frac{1}{a(1+a)}=0 .
$$


Letting $b=a-a^{\prime}$ in (19), we get the expression

$$
\begin{aligned}
\left\langle\left\langle a ; a^{\prime}-a\right]\right. & -\left\langle\left\langle\frac{a}{a^{\prime}} ; 1-\frac{a}{a^{\prime}}\right]\right. \\
& +\left\langle\left\langle\frac{1-a}{1-a^{\prime}} ; 1-\frac{1-a}{1-a^{\prime}}\right]-\left\langle\left\langle\frac{a^{\prime}(1-a)}{a\left(1-a^{\prime}\right)} ; \frac{a^{\prime}}{a}\left(1-\frac{a^{\prime}(1-a)}{a\left(1-a^{\prime}\right)}\right)\right],\right.\right.
\end{aligned}
$$

which can be rewritten in $F_{1} / F_{2}$

$$
\frac{a^{\prime}-a}{a(1-a)}[a]-\frac{a^{\prime}}{a}\left[\frac{a}{a^{\prime}}\right]+\frac{1-a^{\prime}}{1-a}\left[\frac{1-a}{1-a^{\prime}}\right]-\frac{1-a^{\prime}}{1-a}\left[\frac{a^{\prime}(1-a)}{a\left(1-a^{\prime}\right)}\right] .
$$

The proposition will be proved, if we check that the corresponding expression in $\beta(k)$ is 0 . In characteristic not 2 , we have successively in $\beta(k)$, using $a[1 / a]=-[a]$ and $(14)$

$$
\begin{gathered}
\frac{a^{\prime}-a}{a(1-a)}[a]-\frac{a^{\prime}}{a}\left[\frac{a}{a^{\prime}}\right]+\frac{1-a^{\prime}}{1-a}\left[\frac{1-a}{1-a^{\prime}}\right]-\frac{1-a^{\prime}}{1-a}\left[\frac{a^{\prime}(1-a)}{a\left(1-a^{\prime}\right)}\right]= \\
\frac{a^{\prime}-a}{a(1-a)}[a]+\left[\frac{a^{\prime}}{a}\right]-\left[\frac{1-a^{\prime}}{1-a}\right]+\frac{a^{\prime}}{a}\left[\frac{a\left(1-a^{\prime}\right)}{a^{\prime}(1-a)}\right]= \\
\frac{a^{\prime}-a}{a(1-a)}[a]+\frac{a^{\prime}-a}{a}\left[\frac{a}{a-1}\right] .
\end{gathered}
$$

In characteristic 2 , the relation $a[1 / a]=[a]+(1+a) \omega$ introduces an extra term in the first equality, but this term cancels because of the equalities

$$
\begin{gathered}
1+\frac{a^{\prime}}{a}+1+\frac{1+a^{\prime}}{1+a}+\frac{a^{\prime}}{a}\left(1+\frac{a\left(1+a^{\prime}\right.}{a^{\prime}(1+a)}\right)= \\
\left(a+a^{\prime}\right)\left(\frac{1}{a}+\frac{1}{1+a}+\frac{1}{a(1+a)}\right)=0 .
\end{gathered}
$$

Now we are reduced to prove the equality

$$
\left[\frac{a}{a-1}\right]=\frac{1}{a-1}[a] .
$$

In characteristic $\neq 2$

$$
\begin{aligned}
{\left[\frac{a}{a-1}\right]=} & {\left[\left(1-\frac{1}{a}\right)^{-1}\right]=} \\
& \quad-\left(1-\frac{1}{a}\right)^{-1}\left[\left(1-\frac{1}{a}\right)\right]=\frac{a}{1-a}\left[\frac{1}{a}\right]=\frac{1}{a-1}[a] .
\end{aligned}
$$

The relation $\left[\frac{a}{a-1}\right]=\frac{1}{a-1}[a]$ remains true in characteristic 2 , because the extra term in $\omega$ is computed to be

$$
\left(\frac{1}{1+a}+\frac{a}{1+a}+1\right) \omega=2 \omega
$$

TOME $135-2007-\mathrm{N}^{\mathrm{O}} 4$ 
This shows that (20) is zero in $\beta(k)$ and achieves the proof of the proposition.

\subsection{An auxiliary space}

Definition 8.5. - For an arbitrary field $k$, let $\mathcal{J}(k)$ be the $k$-linear space, with generators symbols

$$
\langle a, b\rangle, a, b \in k
$$

and defining relations

$$
\begin{gathered}
\langle a, b\rangle=\langle b, a\rangle, \\
\langle c a, c b\rangle=c\langle a, b\rangle, \\
\langle b, c\rangle-\langle a+b, c\rangle+\langle a, b+c\rangle-\langle a, b\rangle=0,
\end{gathered}
$$

LEMMA 8.6. - If $\operatorname{Char}(k) \neq 2$, we have in $\mathcal{J}(k)$ the relations

(i) $\langle a,-a\rangle=\langle a, 0\rangle=0$,

(ii) $\langle 1-a,-1\rangle=\langle a, 1-a\rangle$.

If $\operatorname{Char}(k)=2$, we have in $\mathcal{J}(k)$ the relation $\langle a, 0\rangle=0$.

Proof. - The equality $\langle a,-a\rangle=0$ is true by (21) and (22) if the characteristic is $\neq 2$. Now the relation (23) for $b=c=0$, gives $\langle a, 0\rangle=0$, since $\langle 0,0\rangle=0$.

To get (ii), we apply (23) to the triple $a, 1-a,-1$.

In characteristic 2 , by (23) applied to the triple $1,1,0$, we get $\langle 1,0\rangle=0$.

Proposition 8.7. - If $\operatorname{Char}(k) \neq 2$, the correspondence

$$
[a] \mapsto\langle a, 1-a\rangle,
$$

induces an isomorphism of $k$-linear spaces

$$
\rho: \beta(k) \rightarrow \mathcal{J}(k),
$$

such that

$$
\rho\left(\sum_{c \in k_{0}}[c]\right)=-\sum_{c \in k_{0}}\langle 1, c\rangle .
$$

Proof. - Note that in this case $[0]=[1]=0$. For the existence of $\rho$, we remark that the assignment $[a] \mapsto\langle a, 1-a\rangle$, is compatible with (15) by the lemma. For the 4-term relation (16), we have to check that for $a \neq 0,1$

$$
\langle a, 1-a\rangle-\left\langle a^{\prime}, 1-a^{\prime}\right\rangle+\left\langle a^{\prime}, a-a^{\prime}\right\rangle+\left\langle 1-a^{\prime}, a^{\prime}-a\right\rangle=0 .
$$

But by the previous lemma

$$
\left\langle a^{\prime}, a-a^{\prime}\right\rangle=a\left\langle\frac{a^{\prime}}{a}, 1-\frac{a^{\prime}}{a}\right\rangle=a\left\langle-1,1-\frac{a^{\prime}}{a}\right\rangle=-\left\langle a, a-a^{\prime}\right\rangle,
$$


and the relation

$$
\langle a, 1-a\rangle-\left\langle a^{\prime}, 1-a^{\prime}\right\rangle+\left\langle 1-a^{\prime}, a^{\prime}-a\right\rangle-\left\langle a, a^{\prime}-a\right\rangle=0,
$$

is (23), applied to the triple: $a, a^{\prime}-a, 1-a^{\prime}$.

Also

$$
\rho\left(\sum_{c \in k_{0}}[c]\right)=\sum_{c \in k_{0}}\langle c, 1-c\rangle=-\sum_{c \in k_{0}}\langle 1,1-c\rangle=-\sum_{c \in k_{0}}\langle 1, c\rangle .
$$

The inverse $\lambda$ of $\rho$ is given on the generators of $\mathcal{J}(k)$ by

$$
\langle a,-a\rangle \mapsto 0,
$$

and

$$
\langle a, b\rangle \mapsto(a+b)\left[\frac{a}{a+b}\right],
$$

for $b \neq-a$. The compatibility of this correspondance with the relation (17) and (18) is clear. We have to check that

$$
\lambda(\langle b, c\rangle)+\lambda(\langle a+b, c\rangle)-\lambda(\langle a, b+c\rangle)+\lambda(\langle a, c\rangle)=0 .
$$

If $b+c, a+b+c$ or $a+b$ is 0 , this stems from (17) and (18). If not, we remark that

$$
(b+c)\left[\frac{b}{b+c}\right]-(a+b+c)\left[\frac{a+b}{a+b+c}\right]+(a+b+c)\left[\frac{a}{a+b+c}\right]-(a+b)\left[\frac{a}{a+b}\right],
$$

can be rewritten

$$
(a+b+c)\left((1-u)\left[\frac{1-u-v}{1-u}\right]-[1-v]+[u]-(1-v)\left[\frac{1-u-v}{1-v}\right]\right)
$$

with

$$
u=\frac{a}{a+b+c}, v=\frac{c}{a+b+c} .
$$

Using (17), we arrive at the expression

$$
(1-u)\left[\frac{v}{1-u}\right]-[1-v]+[u]-(1-v)\left[\frac{u}{1-v}\right],
$$

which is 0 , by (16) and (18).

We note incidentally that the previous expression shows that in characteristic not $2,(8)$ is equivalent to the nice symmetric relation

$$
u\left[\frac{v}{u}\right]+[u]=v\left[\frac{u}{v}\right]+[v] .
$$

The following proposition is due to Sah. 
Proposition 8.8. - If Char $(k)=2$, the correspondence

$$
[a] \mapsto\langle 1,1+a\rangle,
$$

induces an isomorphism of $k$-linear spaces

$$
\rho: \beta(k) \rightarrow \mathcal{J}(k) .
$$

Proof. - By considering (23) for the triple $a+a^{\prime}, 1+a, 1$, we see that the map $\rho$ is well defined. The inverse $\lambda$ comes from the correspondance

$$
\langle a, b\rangle \mapsto a\left[1+\frac{b}{a}\right]
$$

if $a \neq 0$, and

$$
\langle a, b\rangle \mapsto 0
$$

if either $a$ or $b$ is 0 .

The consistency with (22) is trivial. For (21) we need to check that

$$
a\left[1+\frac{b}{a}\right]=b\left[1+\frac{a}{b}\right],
$$

for $a, b \neq 0$. This follows easily from the relation (11) and (12) by letting $c=\frac{a}{b}$.

For consistency with (23), we may assume that $a, b, c$ are all distinct from 0. We can now suppose that $a=1$ by (22). This gives the expression

$$
\langle b, c\rangle+\langle 1+b, c\rangle+\langle 1, b+c\rangle+\langle 1, b\rangle \text {. }
$$

Letting $a=1+b$ and $a^{\prime}=1+b+c$, this expression becomes

$$
\langle 1,1+a\rangle+\left\langle 1,1+a^{\prime}\right\rangle+\left\langle a, a+a^{\prime}\right\rangle+\left\langle 1+a, a+a^{\prime}\right\rangle,
$$

which, for $a \neq 0,1$, is sent in $\beta(k)$ to

$$
[a]+\left[a^{\prime}\right]+a\left[\frac{a^{\prime}}{a}\right]+(1+a)\left[\frac{1+a^{\prime}}{1+a}\right] .
$$

It is sent to

$$
[0]+\left[a^{\prime}\right]+\left[1+a^{\prime}\right]
$$

and

$$
[1]+\left[a^{\prime}\right]+\left[a^{\prime}\right]
$$

for $a=0$ or 1 . All these expressions are 0 in $\beta(k)$.

Proposition 8.9. - Let $\mathcal{J}^{\prime}(k)$ be the quotient of $\mathcal{J}(k)$ by the relation

$$
\sum_{c \in k_{0}}\langle 1, c\rangle=0
$$

when $\operatorname{Char}(k)>0$, and $\mathcal{J}(k)$ otherwise. Consider the map

$$
\begin{aligned}
\psi: k \times k & \rightarrow \mathcal{J}^{\prime}(k) \\
(u, v) & \mapsto\langle u, v\rangle .
\end{aligned}
$$


There exists a map $\phi: k \rightarrow \mathcal{J}^{\prime}(k)$, such that

$$
\begin{aligned}
\psi((a, b)) & =\phi(a+b)-\phi(a)-\phi(b), \\
\phi(a b) & =a \phi(b)+b \phi(a) .
\end{aligned}
$$

Proof. - We first note that the extension $k \supset k_{0}$ of a field over its prime field is always separable, hence by [25] Chap. $11, k$ is smooth over $k_{0}$. The commutative group $\mathcal{C}=k \times \mathcal{J}^{\prime}(k)$ becomes a commutative $\mathbb{Z}$-algebra with unity $(1,0)$, if we define the sum and product by

$$
\begin{aligned}
(a, x)+(b, y) & =(a+b, x+y+\langle a, b\rangle), \\
(a, x)(b, y) & =(a b, a y+b x) .
\end{aligned}
$$

This stems from the defining relations of $\mathcal{J}(k)$. But since in $\mathcal{J}^{\prime}(k)$

$$
n(1,0)=\left(n, \sum_{0<j<n}\langle 1, j\rangle\right),
$$

$\mathcal{C}$ is in fact a $k_{0}$-algebra. We have a commutative diagram of $k_{0}$-algebras

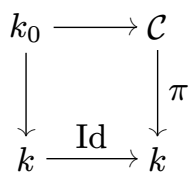

where $\pi$ is the projection. Since the kernel of $\pi$ is a square 0 ideal, by smoothness of $k$ over $k_{0}$ (loc. cit.), $\pi$ has a section $a \mapsto(a, \phi(a))$ in the category of $k_{0}$-algebras. Considering the definitions of the sum and product in $\mathcal{C}$, one gets easily the relations of the proposition.

8.3. The linearized tangent complex. - The cohomology of the linearized tangent complex is given by the the following result.

THEOREM 8.10. - Let $\Omega_{k}^{1}$ be the space of absolute Kähler differentials of degree 1,

(i) If $k$ is of characteristic 0 , the following sequence of $k$-vector spaces is exact

$$
0 \rightarrow \beta(k) \stackrel{f}{\rightarrow} k \otimes k^{\times} \stackrel{g}{\rightarrow} \Omega_{k}^{1} \rightarrow 0,
$$

(ii) If $k$ is of characteristic $>0$, the following sequence of $k$-vector spaces is exact

$$
0 \rightarrow k \stackrel{h}{\rightarrow} \beta(k) \stackrel{f}{\rightarrow} k \otimes k^{\times} \stackrel{g}{\rightarrow} \Omega_{k}^{1} \rightarrow 0 .
$$


In these sequences

$$
\begin{aligned}
f([a]) & =a \otimes a+(1-a) \otimes(1-a), \text { for } a \neq 0,1, \\
g(b \otimes a) & =b \frac{d a}{a} \\
h(1) & =\sum_{c \in k_{0}}[c] .
\end{aligned}
$$

Proof. - One can check that $f$ is well defined, and clearly $g \circ f=0$. If $\operatorname{Char}(k) \neq 0$, we have

$$
f \circ h(1)=\sum_{c \in k_{0}, c \neq 0,1}(c \otimes c+(1-c) \otimes(1-c))=2 \sum_{c \in k_{0}, c \neq 0,1} c \otimes c=0,
$$

because in $k \otimes k^{\times},(-c) \otimes(-c)=-c \otimes c$.

For the exactitude of (24) and (25) in $k \otimes k^{\times}$, we exhibit an inverse to the morphism induced by $g$

$$
k \otimes k^{\times} / \operatorname{Imf} \rightarrow \Omega_{k}^{1}
$$

For this we first remark that $\operatorname{Im} f$ is generated by the elements $a \otimes a+a^{\prime} \otimes a^{\prime}-$ $\left(a+a^{\prime}\right) \otimes\left(a+a^{\prime}\right)\left(a, a^{\prime}, a+a^{\prime} \neq 0,1\right)$, because

$$
\begin{aligned}
f\left(\left(a+a^{\prime}\right)\right. & {\left.\left[\frac{a}{a+a^{\prime}}\right]\right)=} \\
& =\left(a+a^{\prime}\right)\left(\frac{a}{a+a^{\prime}} \otimes \frac{a}{a+a^{\prime}}+\left(1-\frac{a}{a+a^{\prime}}\right) \otimes\left(1-\frac{a}{a+a^{\prime}}\right)\right) \\
& =a \otimes \frac{a}{a+a^{\prime}}+a^{\prime} \otimes \frac{a^{\prime}}{a+a^{\prime}} \\
& =a \otimes a-a \otimes\left(a+a^{\prime}\right)+a^{\prime} \otimes a^{\prime}-a^{\prime} \otimes\left(a+a^{\prime}\right) \\
& =a \otimes a+a^{\prime} \otimes a^{\prime}-\left(a+a^{\prime}\right) \otimes\left(a+a^{\prime}\right) .
\end{aligned}
$$

It is then easy to check that the correspondance $d a \mapsto a \otimes a$ induces the searched inverse.

Now consider the commutative diagram

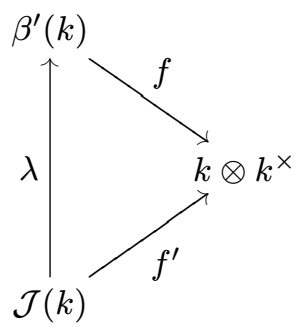

where $f^{\prime}=g \circ \lambda$ is such that, for $a, a^{\prime}$ or $a+a^{\prime}$ equal to 0

$$
f^{\prime}\left(\left\langle a, a^{\prime}\right\rangle\right)=0
$$


and

$$
f^{\prime}\left(\left\langle a, a^{\prime}\right\rangle\right)=a \otimes a+a^{\prime} \otimes a^{\prime}-\left(a+a^{\prime}\right) \otimes\left(a+a^{\prime}\right)
$$

otherwise.

To prove the exactitude in $\beta(k)$ of (24) and (25), we built a retraction of $f^{\prime}$. By Proposition 8.9, there is a map $\phi: k \rightarrow \mathcal{J}(k)$, such that

$$
\begin{aligned}
\phi(a+b)-\phi(a)-\phi(b) & =\langle a, b\rangle, \\
\phi(a b) & =a \phi(b)+b \phi(a) .
\end{aligned}
$$

The map

$$
\begin{aligned}
\nu: k^{\times} & \rightarrow \mathcal{J}(k) \\
a & \mapsto \frac{\phi(a)}{a},
\end{aligned}
$$

satisfies

$$
\nu\left(a a^{\prime}\right)=\nu(a)+\nu\left(a^{\prime}\right) .
$$

Therefore, we have a morphism

$$
r: k \otimes k^{\times} \rightarrow \mathcal{J}(k),
$$

such that

$$
r\left(a \otimes a^{\prime}\right)=-a \nu\left(a^{\prime}\right) .
$$

This is the searched retraction, because for $a, a^{\prime}$ and $a+a^{\prime} \neq 0$,

$$
\begin{aligned}
r \circ f^{\prime}\left(\left\langle a, a^{\prime}\right\rangle\right) & =a \nu(a)+a \nu\left(a^{\prime}\right)-\left(a+a^{\prime}\right) \nu\left(a+a^{\prime}\right) \\
& =\phi\left(a+a^{\prime}\right)-\phi(a)-\phi\left(a^{\prime}\right)=\left\langle a, a^{\prime}\right\rangle .
\end{aligned}
$$

To conclude the proof of the theorem, we have to check that if $\operatorname{Char}(k)=p$, the element $\sum_{c \in k_{0}}[c]$ is not 0 in $\beta(k)$. The argument uses the Witt vectors $W_{2}(k)$ and the idea is due to Sah. We have an abelian extension of additive groups [30]

$$
0 \rightarrow k \rightarrow W_{2}(k) \rightarrow k \rightarrow 0
$$

where the sum in $W_{2}(k)=k \times k$ is given by

$$
\left(a, a^{\prime}\right)+\left(b, b^{\prime}\right)=\left(a+b, a^{\prime}+b^{\prime}+h(a, b)\right),
$$

$h(a, b)=-\sum_{m=1}^{p-1} \frac{1}{p}\left(\begin{array}{c}p \\ m\end{array}\right) a^{m} b^{p-m}$, being the related cocycle. The point is that this cocycle induces a map

$$
\chi: \mathcal{J}(k) \rightarrow k,
$$

with $\chi(\langle a, b\rangle)=h(a, b)$. But by simple calculations

$$
p\left(a, a^{\prime}\right)=\left(0, a \chi\left(\sum_{c \in k_{0}}\langle 1, c\rangle\right)\right) .
$$

TOME $135-2007-\mathrm{N}^{\mathrm{O}} 4$ 
The relation in $\mathcal{J}(k): \sum_{c \in k_{0}}\langle 1, c\rangle=0$, would imply that $W_{2}(k)$ is $p$-torsion. This is definitely not the case since $W_{2}(k)$ contains as a subgroup (in fact as a subalgebra) $W_{2}\left(k_{0}\right)=\mathbb{Z} / p^{2} \mathbb{Z}$.

Corollary 8.11. - For a perfect field of characteristic $p, \beta(k)$ is one dimensional. For a number field, $\beta(k) \cong k \otimes k^{\times}$.

Proof. - One remarks that in the first case, $k \otimes k^{\times}=0$, and in the second one $\Omega_{k}^{1}=0$.

REMARK 8.12. - For $k$ of characteristic $p$, a retraction to the map $h$ of Theorem 8.10 can be obtained in the following way. Consider the $1 \frac{1}{2}$-logarithm of Kontsevich [23]

$$
\begin{aligned}
\mathcal{L}_{1 \frac{1}{2}}: k & \rightarrow k \\
x & \mapsto \sum_{i=1}^{p-1} \frac{x^{i}}{i} .
\end{aligned}
$$

The remarks in loc.cit., show that there is a well defined linear map

$$
\rho: \beta(k) \rightarrow k
$$

such that $\rho([a])=\mathcal{L}_{1 \frac{1}{2}}(a)$. And this map is a retraction of $h$ because of the following classical identities

$$
\sum_{x \in k_{0}} x^{i}=0, \text { if } 1 \leq i \leq p-2,
$$

and

$$
\sum_{x \in k_{0}} x^{p-1}=-1
$$

To be complete, we note the following observation of Sah (see [6]).

Proposition 8.13. - Let $k$ be infinite, and consider $k$ as a module over the affine group Aff $(1, k)$, with the action given by product by inverses of the linear parts, then $\beta(k)$ is isomorphic to the homology group $H_{2}(\operatorname{Aff}(1, k), k)$. 


\section{Appendix: Remarks on the tangent complex to the trilogarithmic complex}

We assume in this appendix that $\operatorname{Char}(k)=0$. We would like the previous calculations to be the beginning of an infinitesimal theory for higher logarithms. The next case to be considered is the trilogarithm, and we address here the tangent complex to the Goncharov 3-logarithmic complex. Unfortunatly, the remarks which follow are not totally convincing, and a general theory of the additive $n$-logarithm would probably require another approach.

Recall that Goncharov $[15,14]$ has introduced analogues of the Bloch-Suslin complex. The one related to the 3-logarithm is a cohomological complex beginning in degree 1 which looks like

$$
\Gamma: \mathcal{B}_{3}(k) \stackrel{\delta}{\rightarrow} \mathcal{B}_{2}(k) \otimes k^{\times} \stackrel{\delta}{\rightarrow} \wedge^{3} k^{\times} .
$$

Here $\mathcal{B}_{2}(k)=\mathcal{B}(k)$. The group $\mathcal{B}_{3}(k)$ is generated by symbols $\{x\}_{3}$, where we assume here that $x \neq 0,1$, and the relations comes from the functional equations of the trilogarithm (loc. cit.). The differentials are defined by

$$
\begin{gathered}
\delta\left(\{x\}_{3}\right)=\{x\}_{2} \otimes x, \\
\delta\left(\{x\}_{2} \otimes y\right)=x \wedge(1-x) \wedge y .
\end{gathered}
$$

Goncharov has given morphisms

$$
H^{i}(\Gamma \otimes \mathbb{Q}) \rightarrow K_{6-i}^{(3)}(k)
$$

for $i=1,2,3$, where the $K_{i}^{(j)}(k)$ are the pieces of the Adams decomposition of the algebraic $K$-theory group $K_{i}(k) \otimes \mathbb{Q}$.

We get a filtration of the tangent group $T \mathcal{B}_{3}(k)$, in the same way as above for $T \mathcal{B}(k)$. Let $\mathcal{B}_{3}^{[i]}$ be the pieces of the associated graded group. We denote here the graded group associated to $T \mathcal{B}_{2}(k)$ by $\oplus_{i=1,2,3} \mathcal{B}_{2}^{[i]}$. It would be nice to have $\mathcal{B}_{3}^{[i]}=0$, for $i>5$. Assume this hypothesis. Calculations show that the graded tangent complex to $\Gamma$ is the sum of the following complexes $\Gamma^{[i]}$, begining in degree 1 , where $\mathcal{B}_{3}^{[i]}$ is generated by multiadditive and symmetric symbols

$$
\begin{aligned}
& \left\langle\left\langle a ; b_{j}, j=1, \ldots, i\right]_{3} .\right. \\
& \Gamma^{[5]}: 0 \rightarrow \mathcal{B}_{3}^{[5]} \rightarrow \\
& \Gamma^{[4]}: 0 \rightarrow \mathcal{B}_{3}^{[4]} \rightarrow \quad \mathcal{B}_{2}^{[3]} \otimes k \quad \rightarrow \quad 0 \\
& \Gamma^{[3]}: 0 \rightarrow \mathcal{B}_{3}^{[3]} \rightarrow\left(\mathcal{B}_{2}^{[2]} \otimes k\right) \oplus\left(\mathcal{B}_{2}^{[3]} \otimes k^{\times}\right) \rightarrow \quad \wedge^{3} k \quad \rightarrow 0 \\
& \Gamma^{[2]}: 0 \rightarrow \mathcal{B}_{3}^{[2]} \rightarrow\left(\mathcal{B}_{2}^{[1]} \otimes k\right) \oplus\left(\mathcal{B}_{2}^{[2]} \otimes k^{\times}\right) \rightarrow \wedge^{2} k \otimes k^{\times} \rightarrow 0 \\
& \Gamma^{[1]}: 0 \rightarrow \mathcal{B}_{3}^{[1]} \rightarrow\left(\mathcal{B}_{2}(k) \otimes k\right) \oplus\left(\mathcal{B}_{2}^{[1]} \otimes k^{\times}\right) \rightarrow k \otimes \wedge^{2} k^{\times} \rightarrow 0
\end{aligned}
$$

with the morphisms defined on generators as follows. 
For weight 4

$$
\left\langle\langle a ; b _ { 1 } , \ldots , b _ { 4 } ] _ { 3 } \mapsto \sum _ { i = 1 } ^ { 4 } \left\langle\left\langle a ; b_{j}, j \neq i\right] \otimes \frac{b_{i}}{a} .\right.\right.
$$

For weight 3

$$
\begin{aligned}
&\left\langle\left\langle a ; b_{1}, b_{2}, b_{3}\right]_{3}\right. \mapsto\left(\sum_{i=1}^{3}\left\langle\left\langle a ; b_{j}, j \neq i\right] \otimes \frac{b_{i}}{a}\right) \oplus\left(\left\langle a ; b_{1}, b_{2}, b_{3}\right] \otimes a\right),\right. \\
&\left\langle\left\langle a ; b_{1}, b_{2}\right] \otimes d\right. \mapsto\left(\frac{b_{1}}{1-a} \wedge \frac{b_{2}}{a}-\frac{b_{1}}{a} \wedge \frac{b_{2}}{1-a}\right) \wedge d, \\
&\left\langle\left\langle a ; b_{1}, b_{2}, b_{3}\right] \mapsto 0 .\right.
\end{aligned}
$$

For weight 2

$$
\begin{aligned}
\left\langle\left\langle a ; b_{1}, b_{2}\right]_{3}\right. & \mapsto\left(\left\langle a ; b_{1}\right] \otimes \frac{b_{2}}{a}+\left\langle\left\langle a ; b_{2}\right] \otimes \frac{b_{1}}{a}\right) \oplus\left\langle\left\langle a ; b_{1}, b_{2}\right] \otimes a,\right.\right. \\
\langle\langle a ; b] \otimes d & \mapsto-\left(\frac{b}{1-a} \wedge d \otimes a+\frac{b}{a} \wedge d \otimes(1-a)\right), \\
\left\langle\left\langle a ; b_{1}, b_{2}\right] \otimes c\right. & \mapsto\left(\frac{b_{1}}{1-a} \wedge \frac{b_{2}}{a}-\frac{b_{1}}{a} \wedge \frac{b_{2}}{1-a}\right) \otimes c .
\end{aligned}
$$

For weight 1

$$
\begin{aligned}
\left\langle\langle a ; b]_{3}\right. & \mapsto\left(-\{a\} \otimes \frac{b}{a}\right) \oplus(\langle\langle a ; b] \otimes a), \\
\{a\} \otimes b & \mapsto b \otimes a \wedge(1-a), \\
\langle\langle a ; b] \otimes c & \mapsto \frac{b}{a} \otimes(1-a) \wedge c+\frac{b}{1-a} \otimes a \wedge c .
\end{aligned}
$$

Taking into account the results of the previous sections, these complexes can be rewritten

$$
\begin{aligned}
& 0 \rightarrow \mathcal{B}_{3}^{[5]} \rightarrow \quad 0 \\
& 0 \rightarrow \mathcal{B}_{3}^{[4]} \rightarrow \quad k \otimes k \quad \rightarrow \quad 0 \\
& 0 \rightarrow \mathcal{B}_{3}^{[3]} \rightarrow\left(\wedge^{2} k \otimes k\right) \oplus\left(k \otimes k^{\times}\right) \rightarrow \wedge^{3} k \quad \rightarrow 0 \\
& 0 \rightarrow \mathcal{B}_{3}^{[2]} \rightarrow(\beta(k) \otimes k) \oplus\left(\wedge^{2} k \otimes k^{\times}\right) \rightarrow \wedge^{2} k \otimes k^{\times} \rightarrow 0 \\
& 0 \rightarrow \mathcal{B}_{3}^{[1]} \rightarrow(\mathcal{B}(k) \otimes k) \oplus\left(\beta(k) \otimes k^{\times}\right) \rightarrow k \otimes \wedge^{2} k^{\times} \rightarrow 0
\end{aligned}
$$

Here are a few results on the cohomology of these complexes.

Proposition 9.1. - (i) $H^{2}\left(\Gamma^{[1]}\right)=0$ and $H^{3}\left(\Gamma^{[1]}\right)=\Omega_{k}^{2}$.

(ii) $H^{3}\left(\Gamma^{[i]}\right)=0$ for $i=2$, 3. If $k$ is quadratically closed, $H^{2}\left(\Gamma^{[4]}\right)=0$. 
Proof. - The weight 1 complex has been explicited in [8] and we refer to this paper for the details. It is straightforward that $H^{3}\left(\Gamma^{[i]}\right)=0$ for $i=2,3$. Letting $b_{1}=b_{2}=b_{3}=b_{4}$, one can see that $H^{2}\left(\Gamma^{[4]}\right)=0$, if $k$ is quadratically closed.

Proposition 9.2. - There is a surjective morphism

$$
H^{2}\left(\Gamma^{[3]}\right) \rightarrow \Omega_{k}^{1}
$$

Proof. - In the complex $\Gamma^{[3]}$

$$
0 \rightarrow \mathcal{B}_{3}^{[3]} \stackrel{f}{\rightarrow}\left(\wedge^{2} k \otimes k\right) \oplus\left(k \otimes k^{\times}\right) \stackrel{g}{\rightarrow} \wedge^{3} k \rightarrow 0,
$$

we have $g=g_{1}+g_{2}$, with

$$
\begin{aligned}
g_{1}(u \wedge v \otimes w) & =u \wedge v \wedge w, \\
g_{2}(b \otimes a) & =0
\end{aligned}
$$

and $f=f_{1}+f_{2}$, with

$$
\begin{gathered}
f_{1}: \mathcal{B}_{3}^{[3]} \rightarrow \wedge^{2} k \otimes k \\
f_{1}\left(\left\langle a ; a(1-a) b_{1}, a(1-a) b_{2}, a(1-a) b_{3}\right]_{3}\right) \\
=\left(a b_{1} \wedge(1-a) b_{2}-(1-a) b_{1} \wedge a b_{2}\right) \otimes(1-a) b_{3} \\
+\left(a b_{1} \wedge(1-a) b_{3}-(1-a) b_{1} \wedge a b_{3}\right) \otimes(1-a) b_{2} \\
+\left(a b_{2} \wedge(1-a) b_{3}-(1-a) b_{2} \wedge a b_{3}\right) \otimes(1-a) b_{1}, \\
f_{2}: \mathcal{B}_{3}^{[3]} \rightarrow k \otimes k^{\times} \\
f_{2}\left(\left\langle a ; a(1-a) b_{1}, a(1-a) b_{2}, a(1-a) b_{3}\right]_{3}\right)=a(1-a) b_{1} b_{2} b_{3} \otimes a .
\end{gathered}
$$

We consider the natural morphisms

$$
\begin{aligned}
h_{1} & : \wedge^{2} k \otimes k \\
u & \wedge v \otimes \Omega_{k}^{1} \\
& \wedge w \frac{1}{6}(u w d v-v w d u),
\end{aligned}
$$

and

$$
\begin{aligned}
h_{2}: k \otimes k^{\times} & \rightarrow \Omega_{k}^{1} \\
b \otimes a & \mapsto b \frac{d a}{a} .
\end{aligned}
$$

Simple calculations show that $h_{1} \circ f_{1}=h_{2} \circ f_{2}$. Indeed, we have

$$
\left.h_{1}\left(a b_{1} \wedge(1-a) b_{2}-(1-a) b_{1} \wedge a b_{2}\right) \otimes(1-a) b_{3}\right)=\frac{1}{3}(1-a) b_{1} b_{2} b_{3} d a,
$$


hence

$$
\begin{aligned}
& h_{1} \circ f_{1}\left(《\left\langle a ; a(1-a) b_{1}, a(1-a) b_{2}, a(1-a) b_{3}\right]_{3}\right)= \\
& \quad(1-a) b_{1} b_{2} b_{3} d a=a(1-a) b_{1} b_{2} b_{3} \frac{d a}{a} .
\end{aligned}
$$

We get a complex, where $h=h_{1}-h_{2}$,

$$
\mathcal{B}_{3}^{[3]} \stackrel{f}{\rightarrow} \operatorname{Ker}\left(g_{1}\right) \oplus\left(k \otimes k^{\times}\right) \stackrel{h}{\rightarrow} \Omega_{k}^{1}
$$

It induces a surjective map $H^{2}\left(\Gamma^{[3]}\right) \rightarrow \Omega_{k}^{1}$.

I do not know if this map is injective. Also one can see that the surjectivity of the map

$$
\begin{aligned}
\mathcal{B}_{3}^{[2]} & \rightarrow \mathcal{B}_{2}^{[1]} \otimes k \\
\left\langle\left\langle a ; b_{1}, b_{2}\right]_{3}\right. & \mapsto\left\langle\left\langle a ; b_{1}\right] \otimes \frac{b_{2}}{a}+\left\langle\left\langle a ; b_{2}\right] \otimes \frac{b_{1}}{a},\right.\right.
\end{aligned}
$$

would imply that $H^{2}\left(\Gamma^{[2]}\right)=0$. But this fact seems unlikely.

By cyclic homology (see [7]) the weight decompositions reduce to

$$
\begin{aligned}
& K_{5}^{(3)}(k[\varepsilon], \varepsilon k)=K_{5}^{(3)[5]}(k[\varepsilon]), \\
& K_{4}^{(3)}(k[\varepsilon], \varepsilon k)=K_{4}^{(3)[3]}(k[\varepsilon]), \\
& K_{3}^{(3)}(k[\varepsilon], \varepsilon k)=K_{3}^{(3)[1]}(k[\varepsilon]),
\end{aligned}
$$

with

$$
K_{5}^{(3)[5]}(k[\varepsilon]) \cong k, \quad K_{4}^{(3)[3]}(k[\varepsilon]) \cong \Omega_{k}^{1}, \quad K_{3}^{(3)[1]}(k[\varepsilon]) \cong \Omega_{k}^{2} .
$$

These groups can be displaided on a table, as follows,

$$
\begin{array}{lcc}
k & & \\
0 & 0 & \\
0 & \Omega_{k}^{1} & 0 \\
0 & 0 & 0 \\
0 & 0 & \Omega_{k}^{2}
\end{array}
$$

The previous results give partial coincidence of this tableau with the cohomology groups of the compexes $\Gamma^{[i]}$. But it seems that the two cohomologies differ. Note that we are limited by the fact that we do not know explicitely all the relations for the symbols $\{x\}_{3}$. One can also conjecture the existence of a morphism

$$
\mathcal{B}_{3}^{[5]} \rightarrow k,
$$


induced by the hypothetical "additive trilogarithm". This additive trilogarithm, defined on $\mathrm{TB}_{3}(k)$, could be such that

$$
\langle a ; a(1-a) b]_{3} \mapsto \frac{1}{5 !} a^{2}(1-a) b^{5} .
$$

Finally for conjectures with a wider point of view, we refer to Goncharov [17].

\section{BIBLIOGRAPHY}

[1] S. BLOCH - "On the tangent functor to Quillen K-theory", in Algebraic $K$ theory, I: Higher K-theories (Proc. Conf., Battelle Memorial Inst., Seattle Wash., 1972), Springer, 1973, p. 205-210. Lecture Notes in Math. Vol. 341.

[2] _ Higher regulators, algebraic K-theory, and zeta functions of elliptic curves, CRM Monograph Series, vol. 11, American Mathematical Society, 2000.

[3] S. Bloch \& H. Esnault - "The additive dilogarithm", Doc. Math. (2003), p. 131-155, Kazuya Kato's fiftieth birthday.

[4] _ "An additive version of higher Chow groups", Ann. Sci. École Norm. Sup. (4) 36 (2003), p. 463-477.

[5] P. CARTier - "Décomposition des polyèdres: le point sur le troisième problème de Hilbert", Astérisque (1986), p. 261-288, Séminaire Bourbaki, vol. $1984 / 85$.

[6] J.-L. CAthelineau - "Sur l'homologie de $\mathrm{SL}_{2}$ à coefficients dans l'action adjointe", Math. Scand. 63 (1988), p. 51-86.

[7] _ " $\lambda$-structures in algebraic $K$-theory and cyclic homology", $K$ Theory 4 (1990/91), p. 591-606.

[8] _ "Remarques sur les différentielles des polylogarithmes uniformes", Ann. Inst. Fourier (Grenoble) 46 (1996), p. 1327-1347.

[9] J. L. DUPONT - Scissors congruences, group homology and characteristic classes, Nankai Tracts in Mathematics, vol. 1, World Scientific Publishing Co. Inc., 2001.

[10] J. L. Dupont \& C. H. SaH - "Scissors congruences. II", J. Pure Appl. Algebra 25 (1982), p. 159-195.

[11] P. Elbaz-Vincent - "The indecomposable $K_{3}$ of rings and homology of $\mathrm{SL}_{2} "$, J. Pure Appl. Algebra 132 (1998), p. 27-71.

[12] _ "Homology of linear groups with coefficients in the adjoint action and $K$-theory", K-Theory 16 (1999), p. 35-50.

[13] P. Elbaz-Vincent \& H. Gangl - "On poly(ana)logs. I", Compositio Math. 130 (2002), p. 161-210. 
[14] A. Goncharov - "Polylogarithms and motivic Galois groups", in Motives (Seattle, WA, 1991), Proc. Sympos. Pure Math., vol. 55, Amer. Math. Soc., 1994, p. 43-96.

[15] _ "Geometry of configurations, polylogarithms, and motivic cohomology", Adv. Math. 114 (1995), p. 197-318.

[16] _ "Volumes of hyperbolic manifolds and mixed Tate motives", $J$. Amer. Math. Soc. 12 (1999), p. 569-618.

[17] _ "Euclidean scissor congruence groups and mixed Tate motives over dual numbers", Math. Res. Lett. 11 (2004), p. 771-784.

[18] T. G. GoodwiLliE - "Relative algebraic $K$-theory and cyclic homology", Ann. of Math. (2) 124 (1986), p. 347-402.

[19] J.-G. GREBET - "Aspects infinitésimaux du troisième problème de Hilbert", Ph.D. Thesis, Univ. de Nice Sophia-Antipolis, 2001.

[20] M. Green \& P. Griffiths - "Formal deformation of Chow groups", in The legacy of Niels Henrik Abel, Springer, 2004, p. 467-509.

[21] _ On the tangent space to the space of algebraic cycles on a smooth algebraic variety, Annals of Mathematics Studies, vol. 157, Princeton University Press, 2005.

[22] W. VAN DeR KAllen - "Le $K_{2}$ des nombres duaux", C. R. Acad. Sci. Paris Sér. A-B 273 (1971), p. A1204-A1207.

[23] M. Kontsevich - "The $1 \frac{1}{2}$-logarithm. Appendix to: "On poly(ana)logs. I" [Compositio Math 130 (2002), no. 2, 161-210] by P. Elbaz-Vincent and H. Gangl", Compositio Math. 130 (2002), p. 211-214.

[24] S. Lichtenbaum - "Groups related to scissors-congruence groups", in Algebraic K-theory and algebraic number theory (Honolulu, HI, 1987), Contemp. Math., vol. 83, Amer. Math. Soc., 1989, p. 151-157.

[25] H. Matsumura - Commutative algebra, W. A. Benjamin, Inc., New York, 1970.

[26] J. PARK - "Regulators on additive higher Chow groups", preprint, 2006.

[27] _ "Algebraic cycles and additive dilogarithm", Int. Math. Res. Not. $\operatorname{IMRN}(2007)$.

[28] K. RÜLling - "Additive Chow groups with higher modulus and the generalized de Rham-Witt complex", J. Algebraic Geom. (2007), p. 109-169.

[29] C. H. SAH - Letter to the author, August 1986.

[30] J-P. Serre - Corps locaux, Hermann, 1968, $2^{\mathrm{e}}$ édition, Publications de l'Université de Nancago, No. VIII.

[31] A. Suslin - " $K_{3}$ of a field and the Bloch group", Proc. Steklov Inst. Math. 4 (1991), p. 217-239. 